For reference, the following erratum corrects the published version of the paper. These errors have been fixed in this arxiv-version (the article starting on page 2 has the corrected expressions).

\title{
Erratum: Evolution of the Carter constant for inspirals into a black hole: Effect of the black hole quadrupole [Phys. Rev. D 75, 124007 (2007)]
}

\author{
Éanna É. Flanagan, Tanja Hinderer
}

In Eqs. (3.16), (3.17), (3.18), (3.24), (3.25) and (3.26) of this paper, the variable $r$ should be replaced everywhere by the variable $\tilde{r}$, and the variable $\theta$ should be replaced everywhere by the variable $\tilde{\theta}$. The definitions of $\tilde{r}$ and $\tilde{\theta}$ are given in Eq. (2.11). These replacements do not affect the any of the subsequent results in the paper.

Also, the right hand side of Eq. (B3) is missing a term $-4 S L_{z} \tilde{r}$ and Eq. (2.24) is missing a factor of $d \varphi / d \tilde{t}$ in front of $Q$.

Some terms are missing in Eqs. (3.18), (3.26) and (3.30) - (3.33). The additional terms in Eqs. (3.18) and (3.26) are

$$
-\frac{8 Q}{15 \tilde{r}^{7}}\left[-75 K^{2}+2 K \tilde{r}(51 \tilde{r} E+50)+8 \tilde{r}^{2}(\tilde{r} E+1)(3 \tilde{r} E+5)\right]
$$

and

$$
\frac{8 Q}{15 p^{2} \tilde{r}^{7}}\left[25 p^{3}(3 p-4 \tilde{r})+p^{2} \tilde{r}^{2}\left(11-51 e^{2}\right)+32 p \tilde{r}^{3}\left(1-e^{2}\right)+6 \tilde{r}^{4}\left(1-e^{2}\right)^{2}\right],
$$

respectively. These result in additional fractional corrections to Eq. (3.30) given by

$$
-\frac{Q}{p^{2}}\left[\frac{1}{2}+\frac{73}{48} e^{2}+\frac{37}{192} e^{4}\right]
$$

and the full expression replacing the $O(Q)$ terms in Eq. (3.30) is then

$$
\langle\dot{K}\rangle=-\frac{64}{5} \frac{\left(1-e^{2}\right)^{3 / 2}}{p^{3}}\left[1+\frac{7 e^{2}}{8}-\frac{Q}{p^{2}}\left\{1+\frac{8}{3} e^{2}+\frac{11}{12} e^{4}+\left(\frac{13}{4}+\frac{841}{96} e^{2}+\frac{449}{192} e^{4}\right) \cos (2 \iota)\right\}\right]+O(S), O\left(S^{2}\right)-\text { terms. }
$$

Equations (3.31), (3.32) and (3.33) contain typos in the $O(S)$ and $O(Q)$ terms, the corrected expressions are given below. We thank P. Komorowski for pointing this out. Equation (3.31) should be replaced by

$$
\begin{gathered}
\langle\dot{p}\rangle=-\frac{64}{5} \frac{\left(1-e^{2}\right)^{3 / 2}}{p^{3}}\left\{1+\frac{7 e^{2}}{8}-\frac{S \cos (\iota)}{96 p^{3 / 2}}\left(1064+1516 e^{2}+475 e^{4}\right)\right. \\
-\frac{Q}{8 p^{2}}\left[14+\frac{149 e^{2}}{12}+\frac{19 e^{4}}{48}+\left(50+\frac{469 e^{2}}{12}+\frac{227 e^{4}}{24}\right) \cos (2 \iota)\right] \\
\left.+\frac{S^{2}}{64 p^{2}}\left(\frac{1}{3}+e^{2}+\frac{e^{4}}{8}\right)[13-\cos (2 \iota)]\right\},
\end{gathered}
$$

Equation (3.32) should be replaced by

$$
\begin{aligned}
\langle\dot{e}\rangle= & -\frac{304}{15} \frac{e\left(1-e^{2}\right)^{3 / 2}}{p^{4}}\left(1+\frac{121 e^{2}}{304}\right)+\frac{S e\left(1-e^{2}\right)^{3 / 2} \cos (\iota)}{5 p^{11 / 2}}\left(1172+932 e^{2}+\frac{1313 e^{4}}{6}\right) \\
& +\frac{Q\left(1-e^{2}\right)^{3 / 2}}{e p^{6}}\left[32+\frac{785 e^{2}}{3}-\frac{219 e^{4}}{2}+13 e^{6}+\left(32+\frac{2195 e^{2}}{3}+251 e^{4}+\frac{218 e^{6}}{3}\right) \cos (2 \iota)\right] \\
& -\frac{S^{2} e\left(1-e^{2}\right)^{3 / 2}}{8 p^{6}}\left(2+3 e^{2}+\frac{e^{4}}{4}\right)[13-\cos (2 \iota)]
\end{aligned}
$$

and the corrected Eq. (3.33) is

$$
\begin{aligned}
\langle i\rangle= & \frac{S \sin (\iota)\left(1-e^{2}\right)^{3 / 2}}{p^{11 / 2}}\left[\frac{244}{15}+\frac{252}{5} e^{2}+\frac{19}{2} e^{4}\right]-\frac{\left(1-e^{2}\right)^{3 / 2} S^{2} \sin (2 \iota)}{240 p^{6}}\left[8+3 e^{2}\left(8+e^{2}\right)\right] \\
& +\frac{Q \cot (\iota)\left(1-e^{2}\right)^{3 / 2}}{60 p^{6}}\left[312+736 e^{2}-83 e^{4}-\left(408+1268 e^{2}+599 e^{4}\right) \cos (2 \iota)\right] .
\end{aligned}
$$




\title{
Evolution of the Carter constant for inspirals into a black hole: effect of the black hole quadrupole
}

\author{
Éanna É. Flanagan ${ }^{1,2}$ and Tanja Hinderer ${ }^{1}$ \\ 1 Center for Radiophysics and Space Research, Cornell University, Ithaca, NY 14853, USA \\ ${ }^{2}$ Laboratory for Elementary Particle Physics, Cornell University, Ithaca, NY 14853, USA
}

(Dated: November 4, 2018)

\begin{abstract}
We analyze the effect of gravitational radiation reaction on generic orbits around a body with an axisymmetric mass quadrupole moment $Q$ to linear order in $Q$, to the leading post-Newtonian order, and to linear order in the mass ratio. This system admits three constants of the motion in absence of radiation reaction: energy, angular momentum along the symmetry axis, and a third constant analogous to the Carter constant. We compute instantaneous and time-averaged rates of change of these three constants. For a point particle orbiting a black hole, Ryan [15] has computed the leading order evolution of the orbit's Carter constant, which is linear in the spin. Our result, when combined with an interaction quadratic in the spin (the coupling of the black hole's spin to its own radiation reaction field), gives the next to leading order evolution. The effect of the quadrupole, like that of the linear spin term, is to circularize eccentric orbits and to drive the orbital plane towards antialignment with the symmetry axis.

In addition we consider a system of two point masses where one body has a single mass multipole or current multipole of order $l$. To linear order in the mass ratio, to linear order in the multipole, and to the leading post-Newtonian order, we show that there does not exist an analog of the Carter constant for such a system (except for the cases of an $l=1$ current moment and an $l=2$ mass moment). Thus, the existence of the Carter constant in Kerr depends on interaction effects between the different multipoles. With mild additional assumptions, this result falsifies the conjecture that all vacuum, axisymmetric spacetimes posess a third constant of the motion for geodesic motion.
\end{abstract}

PACS numbers: $04.25 . \mathrm{Nx}, 04.30 . \mathrm{Db}$

\section{INTRODUCTION AND SUMMARY}

The inspiral of stellar mass compact objects with masses $\mu$ in the range $\mu \sim 1-100 M_{\odot}$ into massive black holes with masses $M \sim 10^{5}-10^{7} M_{\odot}$ is one of the most important sources for the future space-based gravitational wave detector LISA. Observing such events will provide a variety of information: (i) the masses and spins of black holes can be measured to high accuracy $\left(\sim 10^{-4}\right)$; which can constrain the black hole's growth history [1]; (ii) the observations will give a precise test of general relativity in the strong field regime and unambiguously identify whether the central object is a black hole [2]; and (iii) the measured event rate will give insight into the complex stellar dynamics in galactic nuclei [1]. Analogous inspirals may also be interesting for the advanced stages of ground-based detectors: it has been estimated that advanced LIGO could detect up to $\sim 10-30$ inspirals per year of stellar mass compact objects into intermediate mass black holes with masses $M \sim 10^{2}-10^{4} M_{\odot}$ in globular clusters [3]. Detecting these inspirals and extracting information from the datastream will require accurate models of the gravitational waveform as templates for matched filtering. For computing templates, we therefore need a detailed understanding of the how radiation reaction influences the evolution of bound orbits around Kerr black holes [4-7].

There are three dimensionless parameters characterizing inspirals of bodies into black holes:

- the dimensionless spin parameter $a=|\mathbf{S}| / M^{2}$ of the black hole, where $\mathbf{S}$ is the spin.

- the strength of the interaction potential $\epsilon^{2}=$ $G M / r c^{2}$, i.e. the expansion parameter used in postNewtonian (PN) theory.

- the mass ratio $\mu / M$.

For LISA data analysis we will need waveforms that are accurate to all orders in $a$ and $\epsilon^{2}$, and to leading order in $\mu / M$. However, it is useful to have analytic results in the regimes $a \ll 1$ and/or $\epsilon^{2} \ll 1$. Such approximate results can be useful as a check of numerical schemes that compute more accurate waveforms, for scoping out LISA's data analysis requirements [1, 6], and for assessing the accuracy of the leading order in $\mu / M$ or adiabatic approximation [8 -10]. There is substantial literature on such approximate analytic results, and in this paper we will extend some of these results to higher order.

A long standing difficulty in computing the evolution of generic orbits has been the evolution of the orbit's "Carter constant", a constant of motion which governs the orbital shape and inclination. A theoretical prescription now exists for computing Carter constant evolution to all orders in $\epsilon$ and $a$ in the adiabatic limit $\mu \ll M$ 9, 11 13], but it has not yet been implemented numerically. In this paper we focus on computing analytically the evolution of the Carter constant in the regime $a \ll 1$, $\epsilon \ll 1, \mu / M \ll 1$, extending earlier results by Ryan 14, 15.

We next review existing analytical work on the effects of multipole moments on inspiral waveforms. For non- 
spinning point masses, the phase of the $l=2$ piece of the waveform is known to $O\left(\epsilon^{7}\right)$ beyond leading order [16], while spin corrections are not known to such high order. To study the leading order effects of the central body's multipole moments on the inspiral waveform, in the test mass limit $\mu \ll M$, one has to correct both the conservative and dissipative pieces of the forces on the bodies. For the conservative pieces, it suffices to use the Newtonian action for a binary with an additional multipole interaction potential. For the dissipative pieces, the multipole corrections to the fluxes at infinity of the conserved quantities can simply be added to the known PN point mass results. The lowest order spin-orbit coupling effects on the gravitational radiation were first derived by Kidder 17, then extended by Ryan 14, 15], Gergely [18], and Will [19]. Recently, the corrections of $O\left(\epsilon^{2}\right)$ beyond the leading order to the spin-orbit effects on the fluxes were derived [20, 21]. Corrections to the waveform due to the quadrupole - mass monopole interaction were first considered by Poisson 22], who derived the effect on the time averaged energy flux for circular equatorial orbits. Gergely [23] extended this work to generic orbits and computed the radiative instantaneous and time averaged rates of change of energy $E$, magnitude of angular momentum $|\mathbf{L}|$, and the angle $\kappa=\cos ^{-1}(\mathbf{S} \cdot \mathbf{L})$ between the spin $\mathbf{S}$ and orbital angular momentum $\mathbf{L}$. Instead of the Carter constant, Gergely identified the angular average of the magnitude of the orbital angular momentum, $\bar{L}$, as a constant of motion. The fact that to post-2-Newtonian $(2 \mathrm{PN})$ order there is no time averaged secular evolution of the spin allowed Gergely to obtain expressions for $\dot{L}$ and $\dot{\kappa}$ from the quadrupole formula for the evolution of the total angular momentum $\mathbf{J}=\mathbf{L}+\mathbf{S}$. In a different paper, Gergely [18] showed that in addition to the quadrupole, self-interaction spin effects also contribute at $2 \mathrm{PN}$ order, which was seen previously in the black hole perturbation calculations of Shibata et al. 24]. Gergely calculated the effect of this interaction on the instantaneous and time-averaged fluxes of $E$ and $|\mathbf{L}|$ but did not derive the evolution of the third constant of motion.

In this paper, we will re-examine the effects of the quadrupole moment of the black hole and of the leading order spin self interaction. For a black hole, our analysis will thus contain all effects that are quadratic in spin to the leading order in $\epsilon^{2}$ and in $\mu / M$. Our work will extend earlier work by

- Considering generic orbits.

- Using a natural generalization of the Carter-type constant that can be defined for two point particles when one of them has a quadrupole. This facilitates applying our analysis to Kerr inspirals.

- Computing instantaneous as well as time-averaged fluxes for all three constants of motion: energy $E, z$-component of angular momentum $L_{z}$, and Carter-type constant $K$. For most purposes, only time-averaged fluxes are needed as only they are gauge invariant and physically relevant. However, there is one effect for which the time-averaged fluxes are insufficient, namely transient resonances that occur during an inspiral in Kerr in the vicinity of geodesics for which the radial and azimuthal frequencies are commensurate [10,25]. The instantaneous fluxes derived in this paper will be used in [10] for studying the effect of these resonances on the gravitational wave phasing.

We will analyze the effect of gravitational radiation reaction on orbits around a body with an axisymmetric mass quadrupole moment $Q$ to leading order in $Q$, to the leading post-Newtonian order, and to leading order in the mass ratio. With these approximations the adiabatic approximation holds: gravitational radiation reaction takes place over a timescale much longer than the orbital period, so the orbit looks geodesic on short timescales. We follow Ryan's method of computation [14]: First, we calculate the orbital motion in the absence of radiation reaction and the associated constants of motion. Next, we use the leading order radiation reaction accelerations that act on the particle (given by the Burke-Thorne formula [26] augmented by the relevant spin corrections [14]) to compute the evolution of the constants of motion. In the adiabatic limit, the time-averaged rates of change of the constants of motion can be used to infer the secular orbital evolution. Our results show that a mass quadrupole has the same qualitative effect on the evolution as spin: it tends to circularize eccentric orbits and drive the orbital plane towards antialignment with the symmetry axis of the quadrupole.

The relevance of our result to point particles inspiralling into black holes is as follows. The vacuum spacetime geometry around any stationary body is completely characterized by the body's mass multipole moments $I_{L}=I_{a_{1}, a_{2} \ldots a_{l}}$ and current multipole moments $S_{L}=$ $S_{a_{1}, a_{2} \ldots a_{l}}$ [27]. These moments are defined as coefficients in a power series expansion of the metric in the body's local asymptotic rest frame [28]. For nearly Newtonian sources, they are given by integrals over the source as

$$
\begin{aligned}
I_{L} & \equiv I_{a_{1}, \ldots a_{l}}=\int \rho x_{<a_{1}} \ldots x_{a_{l}>d^{3} x} \\
S_{L} & \equiv S_{a_{1}, \ldots a_{l}}=\int \rho x_{p} v_{q} \epsilon_{p q<a_{1}} x_{a_{2}} \ldots x_{a_{l}>} d^{3} x
\end{aligned}
$$

Here $\rho$ is the mass density and $v_{q}$ is the velocity, and " $<$ ... > means "symmetrize and remove all traces". For axisymmetric situations, the tensor multipole moments $I_{L}\left(S_{L}\right)$ contain only a single independent component, conventionally denoted by $I_{l}\left(S_{l}\right)$ [27]. For a Kerr black hole of mass $M$ and spin $\mathbf{S}$, these moments are given by [27]

$$
I_{l}+i S_{l}=M^{l+1}(i a)^{l},
$$

where $a$ is the dimensionless spin parameter defined by $a=|\mathbf{S}| / M^{2}$. Note that $S_{l}=0$ for even $l$ and $I_{l}=0$ for odd $l$. 
Consider now inspirals into an axisymmetric body which has some arbitrary mass and current multipoles $I_{l}$ and $S_{l}$. Then we can consider effects that are linear in $I_{l}$ and $S_{l}$ for each $l$, effects that are quadratic in the multipoles proportional to $I_{l} I_{l^{\prime}}, I_{l} S_{l^{\prime}}, S_{l} S_{l^{\prime}}$, effects that are cubic, etc. For a general body, all these effects can be separated using their scalings, but for a black hole, $I_{l} \propto a^{l}$ for even $l$ and $S_{l} \propto a^{l}$ for odd $l$ [see Eq.(1.3)], so the effects cannot be separated. For example, a physical effect that scales as $O\left(a^{2}\right)$ could be an effect that is quadratic in the spin or linear in the quadrupole; an analysis in Kerr cannot distinguish these two possibilities. For this reason, it is useful to analyze spacetimes that are more general than Kerr, characterized by arbitrary $I_{l}$ and $S_{l}$, as we do in this paper. For recent work on computing exact metrics characterized by sets of moments $I_{l}$ and $S_{l}$, see Refs. [29, 30] and references therein.

The leading order effect of the black hole's multipoles on the inspiral is the $O(a)$ effect computed by Ryan [15]. This $O(a)$ effect depends linearly on the spin $S_{1}$ and is independent of the higher multipoles $S_{l}$ and $I_{l}$ since these all scale as $O\left(a^{2}\right)$ or smaller. In this paper we compute the $O\left(a^{2}\right)$ effect on the inspiral, which includes the leading order linear effect of the black hole's quadrupole (linear in $\left.I_{2} \equiv Q\right)$ and the leading order spin self-interaction (quadratic in $S_{1}$ ).

We next discuss how these $O\left(a^{2}\right)$ effects scale with the post-Newtonian expansion parameter $\epsilon$. Consider first the conservative orbital dynamics. Here it is easy to see that fractional corrections that are linear in $I_{2}$ scale as $O\left(a^{2} \epsilon^{4}\right)$, while those quadratic in $S_{1}$ scale as $O\left(a^{2} \epsilon^{6}\right)$. Thus, the two types of terms cleanly separate. We compute only the leading order, $O\left(a^{2} \epsilon^{4}\right)$, term. For the dissipative contributions to the orbital motion, however, the scalings are different. There are corrections to the radiation reaction acceleration whose fractional magnitudes are $O\left(a^{2} \epsilon^{4}\right)$ from both types of effects linear in $I_{2}$ and quadratic in $S_{1}$. The effects quadratic in $S_{1}$ are due to the backscattering of the radiation off the piece of spacetime curvature due to the black hole's spin. This effect was first pointed out by Shibata et al. [24], who computed the time-averaged energy flux for circular orbits and small inclination angles based on a PN expansion of black hole perturbations. Later, Gergely [18] analyzed this effect on the instantaneous and time-averaged fluxes of energy and magnitude of orbital angular momentum within the PN framework.

The organization of this paper is as follows. In Sec. II] we study the conservative orbital dynamics of two point particles when one particle is endowed with an axisymmetric quadrupole, in the weak field regime, and to leading order in the mass ratio. In Sec. III, we compute the radiation reaction accelerations and the instantaneous and time-averaged fluxes. In order to have all the contributions at $O\left(a^{2} \epsilon^{4}\right)$ for a black hole, we include in our computations of radiation reaction acceleration the interaction that is quadratic in the spin $S_{1}$. The application to black holes in Sec. IV briefly discusses the qualitative predictions of our results and also compares with previous results.

The methods used in this paper can be applied only to the black hole spin (as analyzed by Ryan [14]) and the black hole quadrupole (as analyzed here). We show in Sec. $\mathrm{V}$ that for the higher order mass and current multipole moments taken individually, an analog of the Carter constant cannot be defined to the order of our approximations. We then show that under mild assumptions, this non-existence result can be extended to exact spacetimes, thus falsifying the conjecture that all vacuum axisymmetric spacetimes possess a third constant of geodesic motion.

\section{EFFECT OF AN AXISYMMETRIC MASS QUADRUPOLE ON THE CONSERVATIVE ORBITAL DYNAMICS}

Consider two point particles $m_{1}$ and $m_{2}$ interacting in Newtonian gravity, where $m_{2} \ll m_{1}$ and where the mass $m_{1}$ has a quadrupole moment $Q_{i j}$ which is axisymmetric:

$$
\begin{aligned}
Q_{i j} & =\int d^{3} x \rho(\mathbf{r})\left[x_{i} x_{j}-\frac{1}{3} r^{2} \delta_{i j}\right] \\
& =Q\left(n_{i} n_{j}-\frac{1}{3} \delta_{i j}\right) .
\end{aligned}
$$

For a Kerr black hole of mass $M$ and dimensionless spin parameter $a$ with spin axis along $\mathbf{n}$, the quadrupole scalar is $Q=-M^{3} a^{2}$.

The action describing this system, to leading order in $m_{2} / m_{1}$, is

$$
S=\int d t\left[\frac{1}{2} \mu \mathbf{v}^{2}-\mu \Phi(\mathbf{r})\right],
$$

where $\mathbf{v}=\dot{\mathbf{r}}$ is the velocity, the potential is

$$
\Phi(\mathbf{r})=-\frac{M}{r}-\frac{3}{2 r^{5}} x^{i} x^{j} Q_{i j},
$$

$\mu$ is the reduced mass and $M$ the total mass of the binary, and we are using units with $G=c=1$. We work to linear order in $Q$, to linear order in $m_{2} / m_{1}$, and to leading order in $M / r$. In this regime, the action (2.3) also describes the conservative effect of the black hole's mass quadrupole on bound test particles in Kerr, as discussed in the introduction. We shall assume that the quadrupole $Q_{i j}$ is constant in time. In reality, the quadrupole will evolve due to torques that act to change the orientation of the central body. An estimate based on treating $m_{1}$ as a rigid body in the Newtonian field of $m_{2}$ gives the scaling of the timescale for the quadrupole to evolve compared to the radiation reaction time as (see Appendix I for details)

$$
\frac{T_{\mathrm{evol}}}{T_{\mathrm{rr}}} \sim\left(\frac{m_{1}}{m_{2}}\right)\left(\frac{M}{r}\right)\left(\frac{\bar{S}}{\bar{Q}}\right) \sim\left(\frac{M}{\mu}\right)\left(\frac{M}{r}\right)\left(\frac{1}{a}\right) .
$$


Here, we have denoted the dimensionless spin and quadrupole of the body by $\bar{S}$ and $\bar{Q}$ respectively, and the last relation applies for a Kerr black hole. Since $\mu / M \ll 1$, the first factor in Eq. (2.5) will be large, and since $1 / a \geq 1$ and for the relativistic regime $M / r \sim 1$, the evolution time is long compared to the radiation reaction time. Therefore we can neglect the evolution of the quadrupole at leading order.

This system admits three conserved quantities, the energy

$$
E=\frac{1}{2} \mu \mathbf{v}^{2}+\mu \Phi(\mathbf{r}),
$$

the $z$-component of angular momentum

$$
L_{z}=\mathbf{e}_{z} \cdot(\mu \mathbf{r} \times \mathbf{v}),
$$

and the Carter-type constant

$$
\begin{aligned}
K= & \mu^{2}(\mathbf{r} \times \mathbf{v})^{2}-\frac{2 Q \mu^{2}}{r^{3}}(\mathbf{n} \cdot \mathbf{r})^{2} \\
& +\frac{Q \mu^{2}}{M}\left[(\mathbf{n} \cdot \mathbf{v})^{2}-\frac{1}{2} \mathbf{v}^{2}+\frac{M}{r}\right] .
\end{aligned}
$$

(See below for a derivation of this expression for $K$ ).

\section{A. Conservative orbital dynamics in a Boyer-Lindquist-like coordinate system}

We next specialize to units where $M=1$. We also define the rescaled conserved quantities by $\tilde{E}=E / \mu$, $\tilde{L}_{z}=L_{z} / \mu, \tilde{K}=K / \mu^{2}$, and drop the tildes. These specializations and definitions have the effect of eliminating all factors of $\mu$ and $M$ from the analysis. In spherical polar coordinates $(r, \theta, \varphi)$ the constants of motion $E$ and $L_{z}$ become

$$
\begin{aligned}
E= & \frac{1}{2}\left(\dot{r}^{2}+r^{2} \dot{\theta}^{2}+r^{2} \sin ^{2} \theta \dot{\varphi}^{2}\right)-\frac{1}{r} \\
& +\frac{Q}{2 r^{3}}\left(1-3 \cos ^{2} \theta\right), \\
L_{z}= & r^{2} \sin ^{2} \theta \dot{\varphi} .
\end{aligned}
$$

In these coordinates, the Hamilton-Jacobi equation is not separable, so a separation constant $K$ cannot readily be derived. For this reason we switch to a different coordinate system $(\tilde{r}, \tilde{\theta}, \varphi)$ defined by

$$
\begin{aligned}
& r \cos \theta=\tilde{r} \cos \tilde{\theta}\left(1+\frac{Q}{4 \tilde{r}^{2}}\right), \\
& r \sin \theta=\tilde{r} \sin \tilde{\theta}\left(1-\frac{Q}{4 \tilde{r}^{2}}\right) .
\end{aligned}
$$

We also define a new time variable $\tilde{t}$ by

$$
d t=\left[1-\frac{Q}{2 \tilde{r}^{2}} \cos (2 \tilde{\theta})\right] d \tilde{t} .
$$

The action (2.3) in terms of the new variables to linear order in $Q$ is

$$
\begin{aligned}
S=\int d \tilde{t} & \left\{\frac{1}{2}\left(\frac{d \tilde{r}}{d \tilde{t}}\right)^{2}+\frac{1}{2} \tilde{r}^{2}\left(\frac{d \tilde{\theta}}{d \tilde{t}}\right)^{2}\right. \\
& +\frac{1}{2} \tilde{r}^{2} \sin ^{2} \tilde{\theta}\left(\frac{d \varphi}{d \tilde{t}}\right)^{2}\left[1-\frac{Q}{\tilde{r}^{2}} \sin ^{2} \tilde{\theta}\right] \\
& \left.+\frac{1}{\tilde{r}}+\frac{Q}{4 \tilde{r}^{3}}\right\} .
\end{aligned}
$$

However, a difficulty is that the action (2.13) does not give the same dynamics as the original action (2.3). The reason is that for solutions of the equations of motion for the action (2.3), the variation of the action vanishes for paths with fixed endpoints for which the time interval $\Delta t$ is fixed. Similarly, for solutions of the equations of motion for the action (2.13), the variation of the action vanishes for paths with fixed endpoints for which the time interval $\Delta \tilde{t}$ is fixed. The two sets of varied paths are not the same, since $\Delta t \neq \Delta \tilde{t}$ in general. Therefore, solutions of the Euler-Lagrange equations for the action (2.3) do not correspond to solutions of the Euler-Lagrange equations for the action (2.13). However, in the special case of zeroenergy motions, the extra terms in the variation of the action vanish. Thus, a way around this difficulty is to modify the original action to be

$$
\hat{S}=\int d t\left[\frac{1}{2} \mu \mathbf{v}^{2}-\mu \Phi(\mathbf{r})+E\right] .
$$

This action has the same extrema as the action (2.3), and for motion with physical energy $E$, the energy computed with this action is zero. Transforming to the new variables yields, to linear order in $Q$ :

$$
\begin{aligned}
\hat{S}=\int d \tilde{t} & \left\{\frac{1}{2}\left(\frac{d \tilde{r}}{d \tilde{t}}\right)^{2}+\frac{1}{2} \tilde{r}^{2}\left(\frac{d \tilde{\theta}}{d \tilde{t}}\right)^{2}\right. \\
& +\frac{1}{2} \tilde{r}^{2} \sin ^{2} \tilde{\theta}\left(\frac{d \varphi}{d \tilde{t}}\right)^{2}\left[1-\frac{Q}{\tilde{r}^{2}} \sin ^{2} \tilde{\theta}\right] \\
& \left.+\frac{1}{\tilde{r}}+\frac{Q}{4 \tilde{r}^{3}}+E-\frac{Q E}{2 \tilde{r}^{2}} \cos (2 \tilde{\theta})\right\} .
\end{aligned}
$$

The zero-energy motions for this action coincide with the zero energy motions for the action (2.14). We use this action (2.15) as the foundation for the remainder of our analysis in this section.

The $z$-component of angular momentum in terms of the new variables $(\tilde{r}, \tilde{\theta}, \varphi, \tilde{t})$ is

$$
L_{z}=\tilde{r}^{2} \sin ^{2} \tilde{\theta}\left(\frac{d \varphi}{d \tilde{t}}\right)\left[1-\frac{Q}{\tilde{r}^{2}} \sin ^{2} \tilde{\theta}\right] .
$$

We now transform to the Hamiltonian:

$$
\begin{aligned}
\hat{H}= & \frac{1}{2} p_{\tilde{r}}^{2}-\frac{1}{\tilde{r}}-E-\frac{Q}{4 \tilde{r}^{3}}+\frac{Q L_{z}^{2}}{2 \tilde{r}^{4}} \\
& +\frac{1}{2 \tilde{r}^{2}}\left[p_{\tilde{\theta}}^{2}+\frac{L_{z}^{2}}{\sin ^{2} \tilde{\theta}}+Q E \cos (2 \tilde{\theta})\right]
\end{aligned}
$$


and solve the Hamiltonian Jacobi equation. Denoting the separation constant by $K$ we obtain the following two equations for the $\tilde{r}$ and $\tilde{\theta}$ motions:

$$
\left(\frac{d \tilde{r}}{d \tilde{t}}\right)^{2}=2 E+\frac{2}{\tilde{r}}-\frac{K}{\tilde{r}^{2}}+\frac{Q}{2}\left[\frac{1}{\tilde{r}^{3}}-\frac{2 L_{z}^{2}}{\tilde{r}^{4}}\right],
$$

and

$$
\tilde{r}^{4}\left(\frac{d \tilde{\theta}}{d \tilde{t}}\right)^{2}=K-\frac{L_{z}^{2}}{\sin ^{2} \tilde{\theta}}-Q E \cos (2 \tilde{\theta}) .
$$

Note that the equations of motion (2.18) and (2.19) have the same structure as the equations of motion for Kerr geodesic motion. Using Eqs. (2.18), (2.19) and (2.16) together with the inverse of the transformation (2.11) to linear order in $Q$, we obtain the expression for $K$ in spherical polar coordinates:

$$
\begin{aligned}
K & =r^{4}\left(\dot{\theta}^{2}+\sin ^{2} \theta \dot{\varphi}^{2}\right)+Q(\dot{r} \cos \theta-r \dot{\theta} \sin \theta)^{2}+\frac{Q}{r} \\
& -\frac{Q}{2}\left(\dot{r}^{2}+r^{2} \dot{\theta}^{2}+r^{2} \sin ^{2} \theta \dot{\varphi}^{2}\right)-\frac{2 Q}{r} \cos ^{2} \theta .
\end{aligned}
$$

This is equivalent to the formula (2.8) quoted earlier.

\section{B. Effects linear in spin on the conservative orbital dynamics}

To include the linear in spin effects, we repeat Ryan's analysis [14, 15] (he only gives the final, time averaged fluxes; we will also give the instantaneous fluxes). We can simply add these linear in spin terms to our results because any terms of order $O(S Q)$ will be higher than the order $a^{2}$ to which we are working. The correction to the action (2.3) due to spin-orbit coupling is

$$
S^{\text {spin-orbit }}=\int d t\left[-\frac{2 \mu S n^{i} \epsilon_{i j k} x_{j} \dot{x}_{k}}{r^{3}}\right] .
$$

We will restrict our analysis to the case when the unit vectors $n_{i}$ corresponding to the axisymmetric quadrupole $Q_{i j}$ and to the spin $S_{i}$ coincide, as they do in Kerr.

Including the spin-orbit term in the action (2.3) results in the following modified expressions for $L_{z}$ and $K$ :

$$
L_{z}=\mathbf{n} \cdot(\mu \mathbf{r} \times \mathbf{v})-\frac{2 S}{r^{3}}\left[\mathbf{r}^{2}-(\mathbf{n} \cdot \mathbf{r})^{2}\right],
$$

and

$$
\begin{aligned}
K= & (\mathbf{r} \times \mathbf{v})^{2}-\frac{4 S}{r} \mathbf{n} \cdot(\mathbf{r} \times \mathbf{v})-\frac{2 Q}{r^{3}}(\mathbf{n} \cdot \mathbf{r})^{2} \\
& +Q\left[(\mathbf{n} \cdot \mathbf{v})^{2}-\frac{1}{2} \mathbf{v}^{2}+\frac{1}{r}\right] .
\end{aligned}
$$

In terms of the Boyer-Lindquist like coordinates, the conserved quantities with the linear in spin terms included are:

$$
L_{z}=\tilde{r}^{2} \sin ^{2} \tilde{\theta}\left(\frac{d \varphi}{d \tilde{t}}\right)-\frac{2 S}{r} \sin ^{2} \tilde{\theta}-Q \sin ^{4} \tilde{\theta}\left(\frac{d \varphi}{d \tilde{t}}\right),
$$

$$
\begin{aligned}
K= & r^{4}\left(\dot{\theta}^{2}+\sin ^{2} \theta \dot{\varphi}^{2}\right)-4 S r \sin ^{2} \theta \dot{\varphi} \\
& -\frac{2 Q}{r} \cos ^{2} \theta+Q(\dot{r} \cos \theta-r \dot{\theta} \sin \theta)^{2}+\frac{Q M}{r} \\
& -\frac{Q}{2}\left(\dot{r}^{2}+r^{2} \dot{\theta}^{2}+r^{2} \sin ^{2} \theta \dot{\varphi}^{2}\right) .
\end{aligned}
$$

The equations of motion are

$$
\left(\frac{d \tilde{r}}{d \tilde{t}}\right)^{2}=2 E+\frac{2}{\tilde{r}}-\frac{K}{\tilde{r}^{2}}-\frac{4 S L_{z}}{\tilde{r}^{3}}+\frac{Q}{2}\left[\frac{1}{\tilde{r}^{3}}-\frac{2 L_{z}^{2}}{\tilde{r}^{4}}\right],
$$

and

$$
\tilde{r}^{4}\left(\frac{d \tilde{\theta}}{d \tilde{t}}\right)^{2}=K-\frac{L_{z}^{2}}{\sin ^{2} \tilde{\theta}}-Q E \cos (2 \tilde{\theta}) .
$$

\section{EFFECTS LINEAR IN QUADRUPOLE AND QUADRATIC IN SPIN ON THE EVOLUTION OF THE CONSTANTS OF MOTION}

\section{A. Evaluation of the radiation reaction force}

The relative acceleration of the two bodies can be written as

$$
\mathbf{a}=-\nabla \Phi(\mathbf{r})+\mathbf{a}_{\mathrm{rr}},
$$

where $\mathbf{a}_{\mathrm{rr}}$ is the radiation-reaction acceleration. Combining this with Eqs. (2.6), (2.22) and (2.23) for $E, L_{z}$ and $K$ gives the following formulae for the time derivatives of the conserved quantities:

$$
\begin{aligned}
\dot{E}= & \mathbf{v} \cdot \mathbf{a}_{\mathrm{rr}}, \\
\dot{L}_{z}= & \mathbf{n} \cdot\left(\mathbf{r} \times \mathbf{a}_{\mathrm{rr}}\right) \\
\dot{K}= & 2(\mathbf{r} \times \mathbf{v}) \cdot\left(\mathbf{r} \times \mathbf{a}_{\mathrm{rr}}\right)-\frac{4 S}{r} \mathbf{n} \cdot\left(\mathbf{r} \times \mathbf{a}_{\mathrm{rr}}\right) \\
& +2 Q(\mathbf{n} \cdot \mathbf{v})\left(\mathbf{n} \cdot \mathbf{a}_{\mathrm{rr}}\right)-Q \mathbf{v} \cdot \mathbf{a}_{\mathrm{rr}} .
\end{aligned}
$$

The standard expression for the leading order radiation reaction acceleration acting on one of the bodies is [31]:

$$
\begin{aligned}
a_{\mathrm{rr}}^{j}= & -\frac{2}{5} I_{j k}^{(5)} x_{k}+\frac{16}{45} \epsilon_{j p q} S_{p k}^{(6)} x_{k} x_{q}+\frac{32}{45} \epsilon_{j p q} S_{p k}^{(5)} x_{k} v_{q} \\
& +\frac{32}{45} \epsilon_{p q[j} S_{k] p}^{(5)} x_{q} v_{k} .
\end{aligned}
$$

Here the superscripts in parentheses indicate the number of time derivatives and square brackets on the indices denote antisymmetrization.

The multipole moments $I_{j k}(t)$ and $S_{j k}(t)$ in Eq. (3.5) are the total multipole moments of the spacetime, i.e. approximately those of the black hole plus those due to the orbital motion. The expression (3.5) is formulated in asymptotically Cartesian mass centered (ACMC) coordinates of the system, which are displaced from the coordinates used in Sec. [I] by an amount [28]

$$
\delta \mathbf{r}(t)=-\frac{\mu}{M} \mathbf{r}(t)
$$

This displacement contributes to the radiation reaction acceleration in the following ways: 
1. The black hole multipole moments $I_{l}$ and $S_{l}$, which are time-independent in the coordinates used in Sec. II will be displaced by $\delta \mathbf{r}$ and thus will contribute to the $(l+1)$ th ACMC radiative multipole [28].

2. The constants of motion are defined in terms of the black hole centered coordinates used in Sec. II] so the acceleration $\mathbf{a}_{\mathrm{rr}}$ we need in Eqs. (3.2) - (3.4) is the relative acceleration. This requires calculating the acceleration of both the black hole and the point mass in the ACMC coordinates using (3.5), and then subtracting to find $\mathbf{a}_{\mathrm{rr}}=\mathbf{a}_{\mathrm{rr}}^{\mu}-\mathbf{a}_{\mathrm{rr}}^{M}$ [14]. To leading order in $\mu$, the only effect of the acceleration of the black hole is via a backreaction of the radiation field: the $l$ th black hole moments couple to the $(l+1)$ th radiative moments, thus producing an additional contribution to the acceleration.

For our calculations at $O\left(S_{1} \epsilon^{3}\right), O\left(I_{2} \epsilon^{4}\right), O\left(S_{1}^{2} \epsilon^{4}\right)$, we can make the following simplifications:

- quadrupole corrections: The fractional corrections linear in $I_{2}=Q$ that scale as $O\left(a^{2} \epsilon^{4}\right)$ require only the effect of $I_{2}$ on the conservative orbital dynamics as computed in Sec. IA and the Burke-Thorne formula for the radiation reaction acceleration [given by the first term in Eq. [3.5] ].

- spin-spin corrections: As discussed in the introduction, the fractional corrections quadratic in $S_{1}$ to the conservative dynamics scale as $O\left(a^{2} \epsilon^{6}\right)$ and are subleading order effects which we neglect. At $O\left(a^{2} \epsilon^{4}\right)$, the only effect quadratic in $S_{1}$ is the backscattering of the radiation off the spacetime curvature due to the spin. As discussed in item 1. above, the black hole's current dipole $S_{i}=S_{1} \delta_{i 3}$ (taking the $z$-axis to be the symmetry axis) will contribute to the radiative current quadrupole an amount

$$
S_{i j}^{\mathrm{spin}}=-\frac{3}{2} \frac{\mu}{M} S_{1} x_{i} \delta_{j 3}
$$

The black hole's current dipole $S_{i}$ will couple to the gravitomagnetic radiation field due to $S_{i j}$ as discussed in item 2. above, and contribute to the relative acceleration as [14]:

$$
a_{\mathrm{rr}}^{j \mathrm{spin}}=\frac{8}{15} S_{1} \delta_{i 3} S_{i j}^{(5)}
$$

For our purposes of computing terms quadratic in the spin, we substitute $S_{i j}^{\text {spin }}$ for $S_{i j}$ in Eq. (3.8). Evaluating these quadratic in spin terms requires only the Newtonian conservative dynamics, i.e. the results of Sec. II and Eqs. (3.2) - (3.4) with the quadrupole set to zero.

- linear in spin corrections: Contributions to these effects are from Eq. (3.5) with the current quadrupole replaced by just the spin contribution (3.7), and from Eq. (3.8) evaluated using only the orbital current quadrupole.

With these simplifications, we replace the expression (3.5) for the radiation reaction acceleration with

$$
\begin{aligned}
a_{\mathrm{rr}}^{j}= & -\frac{2}{5} I_{j k}^{(5)} x_{k}+\frac{16}{45} \epsilon_{j p q} S_{p k}^{(6) \text { spin }} x_{k} x_{q} \\
& +\frac{32}{45} \epsilon_{j p q} S_{p k}^{(5) \text { spin }} x_{k} v_{q}+\frac{32}{45} \epsilon_{p q[j} S_{k] p}^{(5) \text { spin }} x_{q} v_{k} \\
& +\frac{8}{15} S_{1} \delta_{i 3}\left[S_{i j}^{(5) \text { orbit }}+S_{i j}^{(5) \text { spin }]}\right.
\end{aligned}
$$

To justify these approximations, consider the scaling of the contribution of black hole's acceleration to the orbital dynamics. The mass and current multipoles of the black hole contribute terms to the Hamiltonian that scale with $\epsilon$ as

$$
\Delta H \sim S_{l} \epsilon^{2 l+3} \& I_{l} \epsilon^{2 l+2}
$$

Since the Newtonian energy scales as $\epsilon^{2}$, the fractional correction to the orbital dynamics scale as

$$
\Delta H / E \sim S_{l} \epsilon^{2 l+1} \& I_{l} \epsilon^{2 l}
$$

To $O\left(\epsilon^{4}\right)$, the only radiative multipole moments that contribute to the acceleration (3.5) are the mass quadrupole $I_{2}$, the mass octupole $I_{3}$, and the current quadrupole $S_{2}$ (cf. [17]). Since we are focusing only on the leading order terms quadratic in spin (these can simply be added to the known $2 \mathrm{PN}$ point particle and $1.5 \mathrm{PN}$ linear in spin results), the only terms in Eq. (3.5) relevant for our purposes are those given in Eq. (3.9). The results from a computation of the fully relativistic metric perturbation for black hole inspirals [24] show that quadratic in spin corrections to the $l=2$ piece compared to the flat space Burke-Thorne formula first appear at $O\left(a^{2} \epsilon^{4}\right)$, which is consistent with the above arguments.

\section{B. Instantaneous fluxes}

We evaluate the radiation reaction force as follows. The total mass and current quadrupole moment of the system are

$$
\begin{aligned}
Q_{i j}^{\mathrm{T}} & =Q_{i j}+\mu x_{i} x_{j}, \\
S_{i j}^{\mathrm{T}} & =S_{i j}^{\mathrm{spin}}+x_{i} \epsilon_{j k m} x_{k} \dot{x}_{m},
\end{aligned}
$$

where from Eq. 2.11

$$
\begin{aligned}
x_{i}= & {\left[\tilde{r} \sin \tilde{\theta}\left(1-\frac{Q}{4 \tilde{r}^{2}}\right) \cos \varphi, \tilde{r} \sin \tilde{\theta}\left(1-\frac{Q}{4 \tilde{r}^{2}}\right) \sin \varphi\right.} \\
& \left.\tilde{r} \cos \tilde{\theta}\left(1+\frac{Q}{4 \tilde{r}^{2}}\right)\right] .
\end{aligned}
$$


Only the second term in Eq. (3.12) contributes to the time derivative of the quadrupole. We differentiate five times by using

$$
\frac{d}{d t}=\left[1+\frac{Q}{2 \tilde{r}^{2}} \cos (2 \tilde{\theta})\right] \frac{d}{d \tilde{t}},
$$

to the order we are working as discussed above. After each differentiation, we eliminate any occurrences of $d \varphi / d \tilde{t}$ using Eq. (2.24), and we eliminate any occurrences of the second order time derivatives $d^{2} \tilde{r} / d \tilde{t}^{2}$ and $d^{2} \tilde{\theta} / d \tilde{t}^{2}$ in favor of first order time derivatives using (the time derivatives of) Eqs. (2.26) and (2.27). For computing the terms linear and quadratic in $S_{1}$, we set the quadrupole $Q$ to zero in all the formulae. We insert the resulting expression into the formula (3.9) for the self-acceleration, and then into Eqs. (3.2) - (3.4). We eliminate $(d \tilde{r} / d \tilde{t})^{2}$, $(d \tilde{\theta} / d \tilde{t})^{2}$, and $(d \varphi / d \tilde{t})$ in favor of $E, L_{z}$, and $K$ using Eqs. (2.24) - (2.27). In the final expressions for the instantaneous fluxes, we keep only terms that are of $O(S)$, $O(Q)$ and $O\left(S^{2}\right)$ and obtain the following results:

$$
\begin{aligned}
\dot{E} & =\frac{160 K}{3 \tilde{r}^{6}}+\frac{64}{3 \tilde{r}^{5}}+\frac{512 E}{15 \tilde{r}^{4}}-\frac{40 K^{2}}{\tilde{r}^{7}}+\frac{272 K E}{5 \tilde{r}^{5}}+\frac{64 E^{2}}{5 \tilde{r}^{3}} \\
& +\frac{S L_{z}}{\tilde{r}^{9}}\left(196 K^{2}+\frac{952}{3} \tilde{r}^{2}-\frac{3668}{5} K \tilde{r}-352 K E \tilde{r}^{2}+\frac{1024}{3} E \tilde{r}^{3}+\frac{128}{5} E^{2} \tilde{r}^{4}\right) \\
& +\frac{2 Q}{\tilde{r}^{9}}\left[-49 K^{2}-169 K L_{z}^{2}+\tilde{r}\left(\frac{532}{5} K+\frac{3307}{15} L_{z}^{2}\right)+2 \tilde{r}^{2}\left(-\frac{20}{3}+47 K E+\frac{548}{5} L_{z}^{2} E\right)-\frac{152}{5} \tilde{r}^{3} E-16 \tilde{r}^{4} E^{2}\right] \\
& +\frac{Q}{\tilde{r}^{9}}\left[\left(-562 K^{2}+\frac{2998}{3} K \tilde{r}-\frac{320}{3} \tilde{r}^{2}+\frac{5072}{5} K E \tilde{r}^{2}-\frac{4048}{15} \tilde{r}^{3} E-160 \tilde{r}^{4} E^{2}\right) \cos (2 \tilde{\theta})\right] \\
& +\frac{Q}{\tilde{r}^{6}} \sin (2 \tilde{\theta})\left(439 K-\frac{926}{3} \tilde{r}-\frac{1528}{5} \tilde{r}^{2} E\right) \dot{\tilde{\theta}} \dot{\tilde{r}} \\
& +\frac{S^{2}}{\tilde{r}^{9}}\left[\left(-K^{2}+\frac{22}{3} K \tilde{r}-\frac{28}{3} \tilde{r}^{2}+\frac{32}{5} K E \tilde{r}^{2}-\frac{236}{15} \tilde{r}^{3} E-\frac{32}{5} \tilde{r}^{4} E^{2}\right) \cos (2 \tilde{\theta})-\tilde{r}^{3} \sin (2 \tilde{\theta})\left(K+\frac{2}{3} \tilde{r}+\frac{8}{5} \tilde{r}^{2} E\right) \dot{\tilde{\theta}} \dot{\tilde{r}}\right] \\
& +\frac{S^{2}}{\tilde{r}^{9}}\left[-49 K^{2}+6 K L_{z}^{2}+2 \tilde{r}\left(63 K-\frac{16}{3} L_{z}^{2}-\frac{98}{3}\right)+\tilde{r}^{2}\left(112 K E-\frac{48}{5} L_{z}^{2} E\right)-\frac{1652}{15} \tilde{r}^{3} E-\frac{224}{5} \tilde{r}^{4} E^{2}\right],
\end{aligned}
$$

$$
\begin{aligned}
\dot{L}_{z} & =\frac{32 L_{z}}{\tilde{r}^{4}}+\frac{144 L_{z} E}{5 \tilde{r}^{3}}-\frac{24 K L_{z}}{\tilde{r}^{5}} \\
& +\frac{S}{\tilde{r}^{7}}\left[-50 K^{2}+240 K L_{z}^{2}+\frac{62}{5} K \tilde{r}-\frac{7376}{15} L_{z}^{2} \tilde{r}+\frac{316}{3} \tilde{r}^{2}+56 K E \tilde{r}^{2}-\frac{1824}{5} E L_{z}^{2} \tilde{r}^{2}+\frac{624}{5} E \tilde{r}^{3}+\frac{128}{5} E^{2} \tilde{r}^{4}\right] \\
& +\frac{S}{\tilde{r}^{7}}\left(50 K^{2}-\frac{62}{5} K \tilde{r}-\frac{316}{3} \tilde{r}^{2}-56 K E \tilde{r}^{2}-\frac{624}{5} E \tilde{r}^{3}-\frac{128}{5} E^{2} \tilde{r}^{4}\right) \cos (2 \tilde{\theta}) \\
& +\frac{S}{\tilde{r}^{4}}\left(-104 K+64 \tilde{r}+64 E \tilde{r}^{2}\right) \sin (2 \tilde{\theta}) \dot{\tilde{r}} \dot{\tilde{\theta}} \\
& +\frac{Q L_{z}}{5 \tilde{r}^{7}}\left[660 E \tilde{r}^{2}+753 \tilde{r}-360 L_{z}^{2}-435 K+\left(1601 \tilde{r}+1512 \tilde{r}^{2} E-1185 K\right) \cos 2 \tilde{\theta}\right]+\frac{174 Q L_{z}}{\tilde{r}^{4}} \sin (2 \tilde{\theta}) \dot{\tilde{r}} \dot{\tilde{\theta}} \\
& +\frac{2 S^{2} L_{z}}{\tilde{r}^{7}}\left[\frac{72}{5} E \tilde{r}^{2}+16 \tilde{r}-9 K\right],
\end{aligned}
$$


and

$$
\begin{aligned}
\dot{K} & =\frac{16 K}{5 \tilde{r}^{5}}\left(20 \tilde{r}+18 \tilde{r}^{2} E-15 K\right) \\
& +\frac{S L_{z}}{\tilde{r}^{7}}\left(280 K^{2}-\frac{14008}{15} K \tilde{r}+\frac{1264}{3} \tilde{r}^{2}+\frac{2496}{5} E \tilde{r}^{3}-\frac{2528}{5} K E \tilde{r}^{2}+\frac{512}{5} E^{2} \tilde{r}^{4}\right) \\
& +\frac{12 Q}{5 \tilde{r}^{7}}\left[-45 K^{2}+\tilde{r} L_{z}^{2}(83+80 \tilde{r} E)-115 K L_{z}^{2}+14 K \tilde{r}(6+5 \tilde{r} E)\right] \\
& +\frac{4 Q}{15 \tilde{r}^{7}} \cos (2 \tilde{\theta})\left(-2175 K^{2}+2975 K \tilde{r}+80 \tilde{r}^{2}+3012 K E \tilde{r}^{2}-112 E \tilde{r}^{3}-168 E^{2} \tilde{r}^{4}\right) \\
& +\frac{2 Q}{15 \tilde{r}^{4}}\left(3075 K-20 \tilde{r}-192 E \tilde{r}^{2}\right) \sin (2 \tilde{\theta}) \dot{\tilde{\theta} \dot{\tilde{r}}} \\
& +\frac{2 S^{2}}{\tilde{r}^{7}}\left[\left(7 K-2 L_{z}^{2}\right)\left(-3 K+\frac{16}{3} \tilde{r}+\frac{24}{5} E \tilde{r}^{2}\right)+K \cos (2 \tilde{\theta})\left(3 K-\frac{16}{3} \tilde{r}-\frac{24}{5} E \tilde{r}^{2}\right)\right] \\
& +\frac{2 S^{2}}{\tilde{r}^{4}} \sin (2 \tilde{\theta})\left(-4 K+\frac{14}{3} \tilde{r}+\frac{16}{5} E \tilde{r}^{2}\right) \dot{\tilde{\theta}} \dot{\tilde{r}}^{.}
\end{aligned}
$$

\section{Alternative set of constants of the motion}

A body in a generic bound orbit in Kerr traces an open ellipse precessing about the hole's spin axis. For stable orbits the motion is confined to a toroidal region whose shape is determined by $E, L_{z}, K$. The motion can equivalently be characterized by the set of constants inclination angle $\iota$, eccentricity $e$, and semi-latus rectum $p$ defined by Hughes 32]. The constants $\iota, p$ and $e$ are defined by $\cos \iota=L_{z} / \sqrt{K}$, and by $\tilde{r}_{ \pm}=p /(1 \pm e)$, where $\tilde{r}_{ \pm}$are the turning points of the radial motion, and $\tilde{r}$ is the Boyer-Lindquist radial coordinate. This parameterization has a simple physical interpretation: in the Newtonian limit of large $p$, the orbit of the particle is an ellipse of eccentricity $e$ and semilatus rectum $p$ on a plane whose inclination angle to the hole's equatorial plane is $\iota$. In the relativistic regime $p \sim M$, this interpretation of the constants $e, p$, and $\iota$ is no longer valid because the orbit is not an ellipse and $\iota$ is not the angle at which the object crosses the equatorial plane (see Ryan [14] for a discussion).

We adopt here analogous definitions of constants of motion $\iota, e$ and $p$, namely

$$
\begin{aligned}
\cos (\iota) & =L_{z} / \sqrt{K}, \\
\frac{p}{1 \pm e} & =\tilde{r}_{ \pm} .
\end{aligned}
$$

Here $K$ is the conserved quantity (2.23) or (2.25), and $\tilde{r}_{ \pm}$ are the turning points of the radial motion using the $\tilde{r}$ coordinate defined by Eq. (2.11), given by the vanishing of the right-hand side of Eq. (2.26).

We now rewrite our results in terms of the new constants of the motion $e, p$ and $\iota$. We can use Eq. (2.26) together with the equations (3.19) and (3.20) to write $E$, $L_{z}$ and $K$ as functions of $p, e$ and $\iota$. To leading order in $Q$ and $S$ we obtain

$$
\begin{gathered}
K=p\left[1-\frac{2 S \cos \iota}{p^{3 / 2}}\left(3+e^{2}\right)-\left(1+e^{2}\right) \frac{2 Q \cos ^{2} \iota}{p^{2}}\right. \\
\left.+\left(3+e^{2}\right) \frac{Q}{4 p^{2}}\right], \\
E=-\frac{\left(1-e^{2}\right)}{2 p}\left[1+\frac{2 S \cos \iota}{p^{3 / 2}}\left(1-e^{2}\right)\right. \\
\left.\quad+\left(1-e^{2}\right) \frac{Q}{p^{2}}\left(\cos ^{2} \iota-\frac{1}{4}\right)\right], \quad(3.22) \\
L_{z}=\sqrt{p} \cos \iota\left[1-\frac{S \cos \iota}{p^{3 / 2}}\left(3+e^{2}\right)-\left(1+e^{2}\right) \frac{Q \cos ^{2} \iota}{p^{2}}\right. \\
\left.+\left(3+e^{2}\right) \frac{Q}{8 p^{2}}\right] .
\end{gathered}
$$

As discussed in the introduction, the effects quadratic in $S$ on the conservative dynamics scale as $O\left(a^{2} \epsilon^{6}\right)$ and thus are not included in this analysis to $O\left(a^{2} \epsilon^{4}\right)$.

Inserting these relations into the expressions (3.16)(3.18) gives, dropping terms of $O(Q S), O\left(Q^{2}\right)$ and $O\left(Q S^{2}\right)$ : 


$$
\begin{aligned}
\dot{E} & \left.=-\frac{8}{15 p^{2} \tilde{r}^{7}}\left[75 p^{4}-100 p^{3} \tilde{r}+p^{2} \tilde{r}^{2}\left(11-51 e^{2}\right)+32 p \tilde{r}^{3}\left(1-e^{2}\right)\right)-6 \tilde{r}^{4}\left(1-e^{2}\right)^{2}\right] \\
& +\frac{4 S \cos \iota}{15 p^{7 / 2} \tilde{r}^{9}}\left[735 p^{6}-2751 p^{5} \tilde{r}+10 p^{4} \tilde{r}^{2}\left(365-6 e^{2}\right)-128 p \tilde{r}^{5}\left(1-e^{2}\right)^{2}-48 \tilde{r}^{6}\left(e^{2}-1\right)^{3}\right] \\
& +\frac{64 S \cos \iota}{15 p^{3 / 2} \tilde{r}^{6}}\left[5 p\left(-23+3 e^{2}\right)-3 \tilde{r}\left(-9+e^{2}+8 e^{4}\right)\right] \\
& -\frac{Q}{15 p^{4} \tilde{r}^{9}}\left[4005 p^{6}-6499 p^{5} \tilde{r}+2 p^{4} \tilde{r}^{2}\left(1577-1977 e^{2}\right)-24 \tilde{r}^{6}\left(1-e^{2}\right)^{3}-32 p^{3} \tilde{r}^{3}\left(8-33 e^{2}\right)+64 p \tilde{r}^{5}\left(1-2 e^{2}+e^{4}\right)\right] \\
& -\frac{Q}{15 p^{4} \tilde{r}^{9}}\left[24 p^{2} \tilde{r}^{4}\left(5-27 e^{2}+22 e^{4}\right)-p \tilde{r}^{3} \sin (2 \tilde{\theta})\left(6585 p^{2}-4630 p \tilde{r}+2292 \tilde{r}^{2}\left(1-e^{2}\right)\right) \dot{\tilde{\theta}} \dot{\tilde{r}}\right] \\
& -\frac{Q}{15 p^{4} \tilde{r}^{9}}\left[2 p^{2} \cos (2 \tilde{\theta})\left(4215 p^{4}-7495 p^{3} \tilde{r}+4 p^{2} \tilde{r}^{2}\left(1151-951 e^{2}\right)-1012 p \tilde{r}^{3}\left(1-e^{2}\right)+300 \tilde{r}^{4}\left(1-2 e^{2}+e^{4}\right)\right)\right] \\
& -\frac{Q}{15 p^{4} \tilde{r}^{9}} \cos (2 \iota)\left[2535 p^{6}-3307 p^{5} \tilde{r}+12 p^{4} \tilde{r}^{2}\left(37-237 e^{2}\right)-48 \tilde{r}^{6}\left(1-e^{2}\right)^{3}+800 p^{3} \tilde{r}^{3}\left(1+e^{2}\right)+128 p \tilde{r}^{5}\left(1-2 e^{2}+e^{4}\right)\right] \\
& +\frac{204 Q}{15 p^{2} \tilde{r}^{5}} \cos (2 \iota)\left(1+2 e^{2}-3 e^{4}\right) \\
& -\frac{2 S^{2}}{15 p^{2} \tilde{r}^{9}}\left[84 \tilde{r}^{4}\left(1-e^{2}\right)^{2}\left(1+e^{2}\right)^{2}+345 p^{4}-905 p^{3} \tilde{r}-413 p \tilde{r}^{3}\left(1-e^{2}\right)+2 p^{2} \tilde{r}^{2}\left(446-201 e^{2}\right)\right] \\
& -\frac{S^{2}}{15 p^{2} \tilde{r}^{9}} \cos (2 \tilde{\theta})\left[15 p^{4}-110 p^{3} \tilde{r}+4 p^{2} \tilde{r}^{2}\left(47-12 e^{2}\right)-118 p \tilde{r}^{3}\left(1-e^{2}\right)+24 \tilde{r}^{4}\left(1-e^{2}\right)^{2}\left(1+e^{2}\right)^{2}\right] \\
& +\frac{S^{2}}{15 \tilde{r}^{9}} \cos (2 \iota)\left[45 p^{2}-80 p \tilde{r}+36 \tilde{r}^{2}\left(1-e^{2}\right)\right]-\frac{S^{2}}{15 p \tilde{r}^{6}} \sin (2 \tilde{\theta}) \dot{\tilde{r} \dot{\tilde{\theta}}}\left[15 p^{2}+10 p \tilde{r}-12 \tilde{r}^{2}\left(1-e^{2}\right)\right]
\end{aligned}
$$

$$
\begin{aligned}
\dot{L}_{z} & =-\frac{8 \cos \iota}{5 \sqrt{p} \tilde{r}^{5}}\left[15 p^{2}-20 p \tilde{r}+9 \tilde{r}^{2}\left(1-e^{2}\right)\right] \\
& +\frac{2 S}{15 p^{2} \tilde{r}^{7}}\left[525 p^{4}-1751 p^{3} \tilde{r}+34 p^{2} \tilde{r}^{2}\left(61-6 e^{2}\right)+12 p \tilde{r}^{3}\left(-69+29 e^{2}\right)+6 \tilde{r}^{4}\left(17+2 e^{2}-19 e^{4}\right)\right] \\
& +\frac{2 S}{15 p^{2} \tilde{r}^{7}}\left[375 p^{4}-93 p^{3} \tilde{r}+468 p \tilde{r}^{3}\left(1-e^{2}\right)-10 p^{2} \tilde{r}^{2}\left(58+21 e^{2}\right)-48 \tilde{r}^{4}\left(1-2 e^{2}+e^{4}\right)\right] \cos (2 \tilde{\theta}) \\
& +\frac{4 S}{15 p^{2} \tilde{r}^{7}}\left[450 p^{4}-922 p^{3} \tilde{r}-60 p \tilde{r}^{3}\left(3+e^{2}\right)-9 p^{2} \tilde{r}^{2}\left(-83+23 e^{2}\right)+27 \tilde{r}^{4}\left(1+2 e^{2}-3 e^{4}\right)\right) \cos (2 \iota) \\
& -\frac{8 S}{p \tilde{r}^{4}}\left[13 p^{2}-8 p \tilde{r}+4 \tilde{r}^{2}\left(1-e^{2}\right)\right] \sin (2 \tilde{\theta}) \dot{\tilde{r}} \dot{\tilde{\theta}} \\
& -\frac{Q \cos \iota}{5 p^{5 / 2} \tilde{r}^{7}}\left[615 p^{4}-753 p^{3} \tilde{r}+15 p^{2} \tilde{r}^{2}\left(19-31 e^{2}\right)+20 p \tilde{r}^{3}\left(1+3 e^{2}\right)+9 \tilde{r}^{4}\left(1-6 e^{2}+5 e^{4}\right)\right] \\
& -\frac{Q \cos \iota}{5 p^{1 / 2} \tilde{r}^{7}} \cos (2 \tilde{\theta})\left(1185 p^{2}-1601 p \tilde{r}+756 \tilde{r}^{2}\left(1-e^{2}\right)\right) \\
& -\frac{2 Q \cos \iota}{5 p^{5 / 2} \tilde{r}^{7}}\left[2 \cos (2 \iota)\left(45 p^{4}-18 \tilde{r}^{4} e^{2}\left(1-e^{2}\right)-45 p^{2} \tilde{r}^{2}\left(1+e^{2}\right)+20 p \tilde{r}^{3}\left(1+e^{2}\right)\right)-435 p^{3} \tilde{r}^{3} \sin (2 \tilde{\theta}) \dot{\tilde{\theta}} \tilde{\tilde{r}}\right] \\
& -\frac{2 S^{2} \cos \iota}{p^{1 / 2} \tilde{r}^{7}}\left[9 p^{2}-16 p \tilde{r}+\frac{36}{5} \tilde{r}^{2}\left(1-e^{2}\right)\right],
\end{aligned}
$$


and

$$
\begin{aligned}
\dot{K} & =\frac{16}{5 \tilde{r}^{5}}\left[20 p \tilde{r}-15 p^{2}-9 \tilde{r}^{2}\left(1-e^{2}\right)\right] \\
& +\frac{8 S \cos \iota}{15 p^{3 / 2} \tilde{r}^{7}}\left[525 p^{4}-1751 p^{3} \tilde{r}+2 p^{2} \tilde{r}^{2}\left(1172-57 e^{2}\right)+12 p \tilde{r}^{3}\left(-99+19 e^{2}\right)-24 \tilde{r}^{4}\left(-11+4 e^{2}+7 e^{4}\right)\right] \\
& +\frac{2 Q}{5 p^{2} \tilde{r}^{7}}\left[-615 p^{4}+753 p^{3} \tilde{r}+30 p^{2} \tilde{r}^{2}\left(17 e^{2}-9\right)+72 \tilde{r}^{4} e^{2}\left(1-e^{2}\right)-40 p \tilde{r}^{3}\left(1+3 e^{2}\right)\right] \\
& +\frac{2 Q}{5 p^{2} \tilde{r}^{7}} \cos (2 \iota)\left[-345 p^{4}+249 p^{3} \tilde{r}-160 p \tilde{r}^{3}\left(1+e^{2}\right)+120 p^{2} \tilde{r}^{2}\left(1+3 e^{2}\right)+36 \tilde{r}^{4}\left(1+2 e^{2}-3 e^{4}\right)\right] \\
& +\frac{2 Q}{15 p^{2} \tilde{r}^{7}}\left[2 \cos (2 \tilde{\theta})\left(2175 p^{4}-2975 p^{3} \tilde{r}-56 p \tilde{r}^{3}\left(1-e^{2}\right)+2 p^{2} \tilde{r}^{2}\left(713-753 e^{2}\right)+42 \tilde{r}^{4}\left(1-2 e^{2}+e^{4}\right)\right)\right] \\
& +\frac{2 Q}{15 p \tilde{r}^{4}} \sin (2 \tilde{\theta})\left(3075 p^{2}-20 p \tilde{r}+96 \tilde{r}^{2}\left(1-e^{2}\right)\right) \dot{\tilde{r}} \dot{\tilde{\theta}} \\
& +\frac{2 S^{2}}{\tilde{r}^{7}}\left\{2\left[-9 p^{2}+16 p \tilde{r}-\frac{36}{5} \tilde{r}^{2}\left(1-e^{2}\right)\right]+(\cos (2 \tilde{\theta})+\cos (2 \iota))\left[3 p^{2}-\frac{16}{3} p \tilde{r}+\frac{12}{5} \tilde{r}^{2}\left(1-e^{2}\right)\right]\right\} \\
& +\frac{4 S^{2}}{p \tilde{r}^{4}} \sin (2 \tilde{\theta}) \dot{\tilde{r}} \dot{\tilde{\theta}}\left[-2 p^{2}+\frac{7}{3} p \tilde{r}-\frac{4}{5} \tilde{r}^{2}\left(1-e^{2}\right)\right] .
\end{aligned}
$$

\section{Time averaged fluxes}

In this section we will compute the infinite timeaverages $\langle\dot{E}\rangle,\left\langle\dot{L}_{z}\right\rangle$ and $\langle\dot{K}\rangle$ of the fluxes. These averages are defined by

$$
\langle\dot{E}\rangle \equiv \lim _{T \rightarrow \infty} \frac{1}{T} \int_{-T / 2}^{T / 2} \dot{E}(t) d t .
$$

These time-averaged fluxes are sufficient to evolve orbits in the adiabatic regime (except for the effect of res- onances) [12, 25]. In Appendix II, we present two different ways of computing the time averages. The first approach is based on decoupling the $\tilde{r}$ and $\tilde{\theta}$ motion using the analog of the Mino time parameter for geodesic motion in Kerr [12]. The second approach uses the explicit Newtonian parameterization of the orbital motion. Both averaging methods give the following results:

$$
\begin{gathered}
\langle\dot{E}\rangle=-\frac{32}{5} \frac{\left(1-e^{2}\right)^{3 / 2}}{p^{5}}\left[1+\frac{73}{24} e^{2}+\frac{37}{96} e^{4}-\frac{S}{p^{3 / 2}}\left(\frac{73}{12}+\frac{823}{24} e^{2}+\frac{949}{32} e^{4}+\frac{491}{192} e^{6}\right) \cos (\iota)\right. \\
-\frac{Q}{p^{2}}\left\{\frac{1}{2}+\frac{85}{32} e^{2}+\frac{349}{128} e^{4}+\frac{107}{384} e^{6}+\left(\frac{11}{4}+\frac{273}{16} e^{2}+\frac{847}{64} e^{4}+\frac{179}{192} e^{6}\right) \cos (2 \iota)\right\} \\
\left.+\frac{S^{2}}{p^{2}}\left\{\frac{13}{192}+\frac{247}{384} e^{2}+\frac{299}{512} e^{4}+\frac{39}{1024} e^{6}-\left(\frac{1}{192}+\frac{19}{384} e^{2}+\frac{23}{512} e^{4}+\frac{3}{1024} e^{6}\right) \cos (2 \iota)\right\}\right],(3.2 \\
\left\langle\dot{L}_{z}\right\rangle=-\frac{32}{5} \frac{\left(1-e^{2}\right)^{3 / 2}}{p^{7 / 2}} \cos \iota\left[1+\frac{7}{8} e^{2}-\frac{S}{2 p^{3 / 2} \cos \iota}\left\{\frac{61}{24}+7 e^{2}+\frac{271}{64} e^{4}+\left(\frac{61}{8}+\frac{91}{4} e^{2}+\frac{461}{64} e^{4}\right) \cos (2 \iota)\right\}\right. \\
-\frac{Q}{16 p^{2}}\left\{-3-\frac{45}{4} e^{2}+\frac{19}{8} e^{4}+\left(45+148 e^{2}+\frac{331}{8} e^{4}\right) \cos (2 \iota)\right\} \\
\left.+\frac{S^{2}}{16 p^{2}}\left\{1+3 e^{2}+\frac{3}{8} e^{4}\right\}\right],
\end{gathered}
$$




$$
\begin{aligned}
\langle\dot{K}\rangle=-\frac{64}{5} \frac{\left(1-e^{2}\right)^{3 / 2}}{p^{3}}[ & 1+\frac{7}{8} e^{2}-\frac{S}{2 p^{3 / 2}}\left(\frac{97}{6}+37 e^{2}+\frac{211}{16} e^{4}\right) \cos (\iota) \\
& -\frac{Q}{p^{2}}\left\{1+\frac{8}{3} e^{2}+\frac{11}{12} e^{4}+\left(\frac{13}{4}+\frac{841}{96} e^{2}+\frac{449}{192} e^{4}\right) \cos (2 \iota)\right\} \\
& \left.+\frac{S^{2}}{p^{2}}\left\{\frac{13}{192}+\frac{13}{64} e^{2}+\frac{13}{512} e^{4}-\left(\frac{1}{192}+\frac{1}{64} e^{2}+\frac{1}{512} e^{4}\right) \cos (2 \iota)\right\}\right] .
\end{aligned}
$$

Using Eqs. (3.21) and (3.23), we obtain from (3.28) - (3.30) the following time averaged rates of change of the orbital elements $e, p, \iota$ :

$$
\begin{gathered}
\langle\dot{p}\rangle=-\frac{64}{5} \frac{\left(1-e^{2}\right)^{3 / 2}}{p^{3}}\left\{1+\frac{7 e^{2}}{8}-\frac{S \cos (\iota)}{96 p^{3 / 2}}\left(1064+1516 e^{2}+475 e^{4}\right)\right. \\
-\frac{Q}{8 p^{2}}\left[14+\frac{149 e^{2}}{12}+\frac{19 e^{4}}{48}+\left(50+\frac{469 e^{2}}{12}+\frac{227 e^{4}}{24}\right) \cos (2 \iota)\right] \\
\left.+\frac{S^{2}}{64 p^{2}}\left(\frac{1}{3}+e^{2}+\frac{e^{4}}{8}\right)[13-\cos (2 \iota)]\right\}, \\
\langle\dot{e}\rangle=-\frac{304}{15} \frac{e\left(1-e^{2}\right)^{3 / 2}}{p^{4}}\left(1+\frac{121 e^{2}}{304}\right)+\frac{S e\left(1-e^{2}\right)^{3 / 2} \cos (\iota)}{5 p^{11 / 2}}\left(1172+932 e^{2}+\frac{1313 e^{4}}{6}\right) \\
+\frac{Q\left(1-e^{2}\right)^{3 / 2}}{e p^{6}}\left[32+\frac{785 e^{2}}{3}-\frac{219 e^{4}}{2}+13 e^{6}+\left(32+\frac{2195 e^{2}}{3}+251 e^{4}+\frac{218 e^{6}}{3}\right) \cos (2 \iota)\right] \\
-\frac{S^{2} e\left(1-e^{2}\right)^{3 / 2}}{8 p^{6}}\left(2+3 e^{2}+\frac{e^{4}}{4}\right)[13-\cos (2 \iota)], \\
\langle i\rangle=\frac{S \sin (\iota)\left(1-e^{2}\right)^{3 / 2}}{p^{11 / 2}}\left[\frac{244}{15}+\frac{252}{5} e^{2}+\frac{19}{2} e^{4}\right]-\frac{\left(1-e^{2}\right)^{3 / 2} S^{2} \sin (2 \iota)}{240 p^{6}}\left[8+3 e^{2}\left(8+e^{2}\right)\right] \\
+\frac{Q \cot (\iota)\left(1-e^{2}\right)^{3 / 2}}{60 p^{6}}\left[312+736 e^{2}-83 e^{4}-\left(408+1268 e^{2}+599 e^{4}\right) \cos (2 \iota)\right] .
\end{gathered}
$$

\section{APPLICATION TO BLACK HOLES}

\section{A. Qualitative discussion of results}

The above results for the fluxes, Eqs. (3.31), (3.32) and (3.33) show that the correction terms at $O\left(a^{2} \epsilon^{4}\right)$ due to the quadrupole have the same type of effect on the evolution as the linear spin correction computed by Ryan: they tend to circularize eccentric orbits and change the angle $\iota$ such as to become antialigned with the symmetry axis of the quadrupole.

The effects of the terms quadratic in spin are qualitatively different. In the expression (3.28) for $\langle\dot{E}\rangle$, the coefficient of $\cos (2 \iota)$ due to the spin self-interaction has the same sign as the quadrupole term, while the terms not involving $\iota$ have the opposite sign. The terms involving $\cos (2 \iota)$ in Eq. (3.30) for $\langle\dot{K}\rangle$ of $O(Q)$ and $O\left(S^{2}\right)$ terms have the same sign, while the terms not involving $\iota$ have the opposite sign. The fractional spin-spin correction to $\left\langle\dot{L}_{z}\right\rangle$, Eq. (3.29), has no $\iota$-dependence, and in expression (3.33) for $\langle i\rangle$, the dependence on $\iota$ of the two effects $O(Q)$ and $O\left(S^{2}\right)$ is different, too. This is not surprising as the $O(Q)$ effects included here are corrections to the conservative orbital dynamics, while the effects of $O\left(S^{2}\right)$ that we included are due to radiation reaction.

\section{B. Comparison with previous results}

The terms linear in the spin in our results for the time averaged fluxes, Eqs. (3.28) - (3.33), agree with those computed by Ryan, Eqs. (14a) - (15c) of [15], and with those given in Eqs. (2.5) - (2.7) of Ref. [33], when we use the transformations to the variables used by Ryan given in Eqs. (2.3) - (2.4) in [33].

Equation (3.28) for the time averaged energy flux agrees with Eq. (3.10) of Gergely [23] and Eq. (4.15) 
of [18] when we use the following transformations:

$$
\begin{aligned}
K= & \bar{L}^{2}\left[1-\frac{Q}{2 \bar{L}^{4}}\left(\bar{A}^{2} \sin ^{2} \kappa \cos \delta-\left(1-\bar{A}^{2}\right) \cos ^{2} \kappa\right)\right] \\
= & \bar{L}^{2}\left[1-\frac{Q}{\bar{L}^{4}} E \cos ^{2} \kappa\right. \\
& \left.\quad-\frac{Q}{2 \bar{L}^{4}}\left(1+2 \bar{L}^{2}\right) \sin ^{2} \kappa \cos \delta\right], \\
\cos \iota= & \cos \kappa\left[1+\frac{Q}{2 \bar{L}^{4}} E \cos ^{2} \kappa\right. \\
\quad & \left.+\frac{Q}{2 \bar{L}^{4}}\left(1+2 \bar{L}^{2}\right) \sin ^{2} \kappa \cos \delta\right], \\
\xi_{0}= & \frac{1}{2}(\delta+\kappa), \\
\xi_{0}= & \left(\psi_{0}-\psi_{i}\right)+\frac{\pi}{2},
\end{aligned}
$$

where $\bar{A}, \bar{L}, \kappa, \delta, \psi_{0}$ and $\psi_{i}$ are the quantities used by Gergely. The first relation here is obtained from the turning points of the radial motion as follows. We compute $\tilde{r}_{ \pm}$in terms of $E$ and $K$ and map these expressions back to $r$ using Eqs. (2.11). The result can then be compared with the turning points in Gergely's variables, Eq. (2.19) of [23], using the fact that $E$ is the same in both cases. Instead of the evolution of the constants of motion $K$ and $L_{z}$, Gergely computes the rates of change of the magnitude $L$ of the orbital angular momentum and of the angle $\kappa$ defined by $\cos \kappa=(\mathbf{L} \cdot \mathbf{S}) / L$. Using the transformations (4.1) - (4.4) and the definition of $\kappa$ we verify that our Eq. (3.29) agrees with the $\left\langle\dot{L}_{z}\right\rangle$ computed using Gergely's Eqs. (3.23) and (3.35) in [23] and Eq. (4.30) of [18].

In the limit of the circular equatorial orbits analyzed by Poisson [22], our Eq. (3.28) agrees with Poisson's Eq. (22) when we use the transformations and specializations:

$$
\begin{aligned}
p & =\frac{1}{v^{2}}\left[1-\frac{Q}{4} v^{4}\right], \\
\iota & =0, \\
e^{2} & =0, \\
\cos \alpha_{A} & =1,
\end{aligned}
$$

where $v$ and $\alpha_{A}$ are the variables used by Poisson and the relation (4.5) is obtained by comparing the expressions for the constants of motion in the two sets of variables.

The main improvement of our analysis over Gergely's is that we express the results in terms of the Carter-type constant $K$, which facilitates comparing our results with other analyses of black hole inspirals. Our computations also include the spin curvature scattering effects for all three constants of motion; Gergely [18] only considers these effects for two of them: the energy and magnitude of angular momentum, not for the third conserved quantity.

When we expand Eq. (3.28) for small inclination angles and specialize to circular orbits, then after converting $p$ to the parameter $v$ using Eq. (4.5), we obtain

$$
\begin{aligned}
\langle\dot{E}\rangle & =-\frac{32}{5 p^{5}}\left[1-\frac{1}{p^{2}}\left(2 Q+\frac{S^{2}}{16}\right)+\frac{\iota^{2}}{2 p^{2}}\left(11 Q-\frac{S^{2}}{48}\right)\right] \\
& =-\frac{32}{5 p^{5}}\left[1-\frac{a^{2} v^{4}}{16}\left\{33-\frac{527}{6} \iota^{2}\right\}\right] .
\end{aligned}
$$

This result agrees with the terms at $O\left(a^{2} v^{4}\right)$ of Eq. (3.13) of Shibata et al. 24], whose calculations were based on the fully relativistic expressions. This agreement is a check that we have taken into account all the contributions at $O\left(a^{2} \epsilon^{4}\right)$. The analysis in Ref. 24] could not distinguish between effects due to the quadrupole and those due curvature scattering, but we can see from Eq. (4.9) that those two interactions have the opposite dependence on $\iota$. Comparing (4.9) with Eq. (3.7) of [24] (which gives the fluxes into the different modes $(l=2, m, n)$, where $m$ and $n$ are the multiples of the $\varphi$ and $\theta$ frequencies), we see that the terms in the $(2, \pm 2,0)$ and the $(2, \pm 1, \pm 1)$ modes are entirely due to the quadrupole, while the spin-spin interaction effects are fully contained in the $(2, \pm 1,0)$ and $(2,0, \pm 1)$ modes.

\section{NON-EXISTENCE OF A CARTER-TYPE CONSTANT FOR HIGHER MULTIPOLES}

In this section, we show that for a single axisymmetric multipole interaction, it is not possible to find an ana$\log$ of the Carter constant (a conserved quantity which does not correspond to a symmetry of the Lagrangian), except for the cases of spin (treated by Ryan 15]) and mass quadrupole moment (treated in this paper). Our proof is valid only in the approximations in which we work - expanding to linear order in the mass ratio, to the leading post-Newtonian order, and to linear order in the multipole. However we will show below that with very mild additional smoothness assumptions, our nonexistence result extends to exact geodesic motion in exact vacuum spacetimes.

We start in Sec. VA by showing that there is no coordinate system in which the Hamilton-Jacobi equation is separable. Now separability of the Hamilton-Jacobi equation is a sufficient but not a necessary condition for the existence of a additional conserved quantity. Hence, this result does not yield information about the existence or non-existence of an additional constant. Nevertheless we find it to be a suggestive result. Our actual derivation of the non-existence is based on Poisson bracket computations, and is given in Sec. $\mathrm{VB}$.

\section{A. Separability analysis}

Consider a binary of two point masses $m_{1}$ and $m_{2}$, where the mass $m_{1}$ is endowed with a single axisymmetric current multipole moment $S_{l}$ or axisymmetric mass multipole moment $I_{l}$. In this section, we show that the 
Hamilton-Jacobi equation for this motion, to linear order in the multipoles, to linear order in the mass ratio and to the leading post-Newtonian order, is separable only for the cases $S_{1}$ and $I_{2}$.

We choose the symmetry axis to be the $z$-axis and write the action for a general multipole as

$$
\begin{aligned}
S=\int d t \quad\left[\frac{1}{2}\left(\dot{r}^{2}+r^{2} \dot{\theta}^{2}+r^{2} \sin ^{2} \theta \dot{\varphi}^{2}\right)+\frac{1}{r}\right. \\
+f(r, \theta)+g(r, \theta) \dot{\varphi}+E] .
\end{aligned}
$$

For mass moments, $g(r, \theta)=0$, while for current moments $f(r, \theta)=0$. For an axisymmetric multipole of order $l$, the functions $f$ and $g$ will be of the form

$$
f(r, \theta)=\frac{c_{l} I_{l} P_{l}(\cos \theta)}{r^{l+1}}, g(r, \theta)=\frac{d_{l} S_{l} \sin \theta \partial_{\theta} P_{l}(\cos \theta)}{r^{l}},
$$

where $P_{l}(\cos \theta)$ are the Legendre polynomials and $c_{l}$ and $d_{l}$ are constants. We will work to linear order in $f$ and $g$. In Eq. (5.1), we have added the energy term needed when doing a change of time variables, cf. the discussion before Eq. (2.14) in section III. Since $\varphi$ is a cyclic coordinate, $p_{\varphi}=L_{z}$ is a constant of motion and the system has effectively only two degrees of freedom. Note that in the case of a current moment, there will be correction term in $L_{z}$ :

$$
L_{z}=r^{2} \sin ^{2} \theta \dot{\varphi}+g(r, \theta) .
$$

Next, we switch to a different coordinate system $(\tilde{r}, \tilde{\theta}, \varphi)$ defined by

$$
\begin{aligned}
& r=\tilde{r}+\alpha\left(\tilde{r}, \tilde{\theta}, L_{z}\right), \\
& \theta=\tilde{\theta}+\beta\left(\tilde{r}, \tilde{\theta}, L_{z}\right),
\end{aligned}
$$

where the functions $\alpha$ and $\beta$ are yet undetermined. We also define a new time variable $\tilde{t}$ by

$$
d t=\left[1+\gamma\left(\tilde{r}, \tilde{\theta}, L_{z}\right)\right] d \tilde{t}
$$

Since we work to linear order in $f$ and $g$, we can work to linear order in $\alpha, \beta$, and $\gamma$. We then compute the action in the new coordinates and drop the tildes. The Hamiltonian is given by

$$
\begin{aligned}
H= & \frac{1}{2} p_{r}^{2}\left(1+\gamma-2 \alpha_{, r}\right)+\frac{p_{\theta}^{2}}{2 r^{2}}\left(1-\frac{2 \alpha}{r}-2 \beta_{, \theta}+\gamma\right) \\
& +\frac{p_{r} p_{\theta}}{r^{2}}\left(-\alpha, \theta-r^{2} \beta_{, r}\right)-E(1+\gamma) \\
& +\frac{L_{z}^{2}}{2 r^{2} \sin ^{2} \theta}\left(1+\gamma-\frac{2 \alpha}{r}-2 \beta \cot \theta\right) \\
& -\frac{1}{r}\left(1-\frac{\alpha}{r}+\gamma\right)-f-\frac{g L_{z}}{r^{2} \sin ^{2} \theta}
\end{aligned}
$$

and the corresponding Hamilton-Jacobi equation is

$$
\begin{aligned}
0= & \left(\frac{\partial W}{\partial r}\right)^{2} \hat{C}_{1}+\left(\frac{\partial W}{\partial \theta}\right)^{2} \frac{\hat{C}_{2}}{r^{2}} \\
& +2\left(\frac{\partial W}{\partial r}\right)\left(\frac{\partial W}{\partial \theta}\right) \frac{\hat{C}_{3}}{r^{2}}+2 \hat{V}
\end{aligned}
$$

where we have denoted

$$
\begin{aligned}
\hat{C}_{1}= & J(r, \theta)\left[1+\gamma-2 \alpha_{, r}\right]=1+\gamma-2 \alpha_{, r}+j, \\
\hat{C}_{2}= & J(r, \theta)\left[1-\frac{2 \alpha}{r}-2 \beta_{, \theta}+\gamma\right] \\
= & 1-\frac{2 \alpha}{r}-2 \beta_{, \theta}+\gamma+j, \\
\hat{C}_{3}= & J(r, \theta)\left[-\alpha, \theta-r^{2} \beta_{, r}\right]=-\alpha_{, \theta}-r^{2} \beta_{, r}, \quad(5) \\
\hat{V}= & J(r, \theta)\left[\frac{L_{z}^{2}}{2 r^{2} \sin ^{2} \theta}\left(1+\gamma-\frac{2 \alpha}{r}-2 \beta \cot \theta\right)\right. \\
& -\frac{1}{r}\left(1-\frac{\alpha}{r}+\gamma\right)-E(1+\gamma) \\
& \left.-f-\frac{g L_{z}}{r^{2} \sin ^{2} \theta}\right] \\
= & \frac{L_{z}^{2}}{2 r^{2} \sin ^{2} \theta}\left(1+\gamma-\frac{2 \alpha}{r}-2 \beta \cot \theta+j\right) \\
& -E(1+\gamma+j)-\frac{1}{r}\left(1-\frac{\alpha}{r}+\gamma+j\right) \\
& -f-\frac{g L_{z}}{r^{2} \sin ^{2} \theta} .
\end{aligned}
$$

The unperturbed problem is separable, so make the perturbed problem separable, we have multiplied the Hamilton-Jacobi equation by an arbitrary function $J(r, \theta)$, which can be expanded as $J(r, \theta)=1+j(r, \theta)$, where $j(r, \theta)$ is a small perturbation.

To find a solution of the form $W=W_{r}(r)+W_{\theta}(\theta)$, we first specialize to the case where $\hat{C}_{3}=0$ :

$$
-\hat{C}_{3}=\beta_{, r} r^{2}+\alpha_{, \theta}=0 \text {. }
$$

We differentiate Eq. (5.8) with respect to $\theta$, using Eq. (5.8) to write $\left(d W_{r} / d r\right)^{2}$ in terms of $\left(d W_{\theta} / d \theta\right)^{2}$ and then differentiate the result with respect to $r$ to obtain

$$
\begin{aligned}
0= & \left(\frac{d W_{\theta}}{d \theta}\right)^{2} \partial_{r}\left[\frac{\partial_{\theta} \hat{C}_{2}}{\hat{C}_{2}}-\frac{\partial_{\theta} \hat{C}_{1}}{\hat{C}_{1}}\right] \\
& +2 \partial_{r}\left[r^{2} \frac{\partial_{\theta} \hat{V}}{\hat{C}_{2}}-\frac{r^{2} \hat{V} \partial_{\theta} \hat{C}_{1}}{\hat{C}_{1} \hat{C}_{2}}\right] .
\end{aligned}
$$

Expanding Eq. (5.14) to linear order in the small quantities then yields the two conditions for the kinetic and the potential part of the Hamiltonian to be separable:

$$
\begin{aligned}
0= & \partial_{r} \partial_{\theta}\left(2 \alpha_{, r}-\frac{2 \alpha}{r}-2 \beta, \theta\right) \\
0= & \frac{L_{z}^{2}}{\sin ^{2} \theta}\left(2 \beta_{, r} \cot ^{2} \theta-3 \beta_{, r \theta} \cot \theta+\beta_{, r} \csc ^{2} \theta\right) \\
& +\frac{L_{z}^{2}}{\sin ^{2} \theta} \partial_{r}\left[-\frac{\alpha, \theta}{r}+\alpha_{, r \theta}\right] \\
& -\partial_{r} \partial_{\theta}\left[\frac{c_{l} I_{l}}{r^{l-1}} P_{l}(\cos \theta)+\frac{d_{l} S_{l} L_{z}}{r^{l} \sin \theta} \partial_{\theta} P_{l}(\cos \theta)\right] \\
& -\partial_{r}\left[r\left(2 \alpha_{, r \theta}-\frac{\alpha, \theta}{r}\right)+2 E r^{2} \alpha_{, r \theta}\right]
\end{aligned}
$$


where we have used Eq. (5.2) for $f$ and $g$. Therefore, the following conditions must be satisfied:

$$
\begin{aligned}
M_{4}(\theta)-N(r)= & \frac{\alpha}{r}+\beta, \theta-2 \alpha_{, r} \\
M_{1}(\theta)= & 2 \beta \cot ^{2} \theta+\beta \csc ^{2} \theta+\beta_{, \theta \theta} \\
& -3 \beta, \theta \cot \theta \\
M_{2}(\theta)= & r^{2} \partial_{r}\left(r^{2} \beta_{, r}\right) \\
M_{3}(\theta)= & 2 r \alpha_{, r \theta}-\alpha_{, \theta}+\frac{I_{l}}{r^{l-1}} \partial_{\theta} P_{l}(\cos \theta) \\
& -\frac{S_{l} L_{z}}{r^{l}} \partial_{\theta}\left(\csc \theta \partial_{\theta} P_{l}(\cos \theta)\right) .
\end{aligned}
$$

Here, the functions $M$ and $N$ are arbitrary integration constants.

Solving the condition for the kinetic term to be separable, Eq. (5.17), together with Eq. (5.13) gives the general solution that goes to zero at large $r$ as

$$
\begin{aligned}
& \alpha=\frac{A}{r^{n-1}} \cos (n \theta+\nu), \\
& \beta=-\frac{A}{r^{n}} \sin (n \theta+\nu),
\end{aligned}
$$

where $A$ and $\nu$ are arbitrary and $n$ is an integer. These functions must satisfy the conditions (5.18) - (5.20) in order for the potential term to be separable as well. To see when this will be the case, we start by considering Eq. (5.20). Substituting the general ansatz $\alpha=a_{1}(r) a_{2}(\theta)$ shows that $a_{2}^{\prime}=P_{l}^{\prime}$ or $a_{2}^{\prime}=\left(\csc \theta P_{l}^{\prime}\right)^{\prime}$ depending on whether a mass or a current multipole is present. The function $a_{1}(r)$ is then determined from

$$
0=2 r a_{1}^{\prime}-a_{1}+\left\{\begin{array}{l}
c_{l} I_{l} / r^{(l-1)} \\
d_{l} S_{l} L_{z} / r^{l}
\end{array}\right.
$$

Hence,

$$
a_{1}=\left\{\begin{array}{l}
{\left[c_{l} I_{l} /(2 l)\right] r^{(1-l)}} \\
{\left[d_{l} S_{l} L_{z} /(2 l+1)\right] r^{-l}}
\end{array}\right.
$$

so that we obtain for mass moments

$$
\alpha=\frac{c_{l} I_{l}}{2 l} \frac{P_{l}(\cos \theta)}{r^{l-1}}, \quad \beta=\frac{c_{l} I_{l}}{2 l^{2}} \frac{P_{l}^{\prime}(\cos \theta)}{r^{l}}
$$

and for current moments

$$
\begin{aligned}
\alpha & =\frac{d_{l} S_{l} L_{z}}{2 l+1} \frac{\csc \theta P_{l}^{\prime}(\cos \theta)}{r^{l}} \\
\beta & =\frac{d_{l} S_{l} L_{z}}{(2 l+1)(l+1)} \frac{\left(\csc \theta P_{l}^{\prime}(\cos \theta)\right)^{\prime}}{r^{l+1}}
\end{aligned}
$$

where we have used the condition (5.13) to solve for $\beta$.

Substituting this in Eq. (5.19) determines that $l=2$ for mass moments and $l+1=2$ for current moments. For an $l=2$ mass moment, conditions (5.17) and (5.18) are satisfied as well, with $n=2$ and $\nu=0$. For the case of an $l=1$ current moment, the extra term in $H$ is independent of $\theta$ anyway. But for any other multipole interaction, the Hamilton-Jacobi equation will not be separable. For example, for the current octupole $S_{i j k}$, the last term in Eq. (5.7) is proportional to $S_{3} L_{z}\left(5 \cos ^{2} \theta-1\right) / r^{5}$ and is therefore not separable. From Eq. (5.2) one can see that, for a general multipole, the functions $f$ or $g$ contain different powers of $\cos \theta$ appearing with the same power of $r$ since the Legendre polynomials can be expanded as 34]:

$$
P_{l}(\cos \theta)=\sum_{n=0}^{N} \frac{(-1)^{n}(2 l-2 n) !}{2^{l} n !(l-n) !(l-2 n) !}(\cos \theta)^{l-2 n},
$$

where $N=l / 2$ for even $l$ and $N=(l+1) / 2$ for odd $l$. It will not be possible to cancel all of these terms with (5.21) - (5.22) for $l>2$.

The case when $\hat{C}_{3}$ is non-vanishing will only be separable if all the coefficients are functions of $r$ or of $\theta$ only, and if in addition, the potential also depends only on $r$ or on $\theta$. Achieving this for our problem will not be possible because the potential cannot be transformed to the form required for separability.

\section{B. Derivation of non-existence of additional constants of the motion}

In this subsection, we show using Poisson brackets that for a single axisymmetric multipole interaction, to linear order in the multipole and the mass ratio, a first integral analogous to the Carter constant does not exist, except for the cases of mass quadrupole and spin.

Suppose that such a constant does exist. We write the Hamiltonian corresponding to the action (5.1) as $H=$ $H_{0}+\delta H$ and the Carter-type constant as $K=K_{0}+$ $\delta K\left(p_{r}, p_{\theta}, L_{z}, r, \theta\right)$, where

$$
\begin{aligned}
H_{0} & =\frac{p_{r}^{2}}{2}+\frac{p_{\theta}^{2}}{2 r^{2}}+\frac{L_{z}^{2}}{2 r^{2} \sin ^{2} \theta}-\frac{1}{r} \\
\delta H & =-\frac{c_{l} I_{l}}{r^{l+1}} P_{l}(\cos \theta)-\frac{d_{l} S_{l} L_{z}}{r^{l+2} \sin \theta} \partial_{\theta} P_{l}(\cos \theta) \\
K_{0} & =p_{\theta}^{2}+\frac{L_{z}^{2}}{\sin ^{2} \theta}
\end{aligned}
$$

Computing the Poisson bracket gives, to linear order in the perturbations

$$
\begin{aligned}
0 & =\left\{H_{0}, \delta K\right\}+\left\{\delta H, K_{0}\right\} \\
& =\frac{d}{d t} \delta K+\left\{\delta H, K_{0}\right\},
\end{aligned}
$$

where we have used that $\left\{H_{0}, K_{0}\right\}=0$ and the fact that $\left\{H_{0}, \delta K\right\}=d(\delta K) / d t$. Here, $d / d t$ denotes the total time derivative along an orbit $\left(r(t), \theta(t), p_{r}(t), p_{\theta}(t)\right)$ of $H_{0}$ in phase space. The partial differential equation (5.32a) for $\delta K$ thus reduces to a set of ordinary differential equations that can be integrated along the individual orbits in phase space. 
The unperturbed motion for a bound orbit is in a plane, so we can switch from spherical to plane polar coordinates $(r, \psi)$. In terms of these coordinates, we have $H_{0}=p_{r}^{2} / 2+p_{\psi}^{2} / 2, K_{0}=p_{\psi}^{2}$, and $\cos \theta=\sin \iota \sin \left(\psi+\psi_{0}\right)$, with $\cos \iota=L_{z} / \sqrt{K}$ and the constant $\psi_{0}$ denoting the angle between the direction of the periastron and the intersection between the orbital and equatorial plane. Then Eq. (5.32) becomes

$$
\begin{aligned}
\frac{d}{d t} \delta K= & \eta(t), \\
\eta(t)= & -\frac{2 p_{\psi} d_{l} S_{l} L_{z}}{\sin \iota r^{l+2}(t)} \partial_{\psi}\left(\frac{\partial_{\psi} P_{l}\left(\sin \iota \sin \left(\psi(t)+\psi_{0}\right)\right)}{\cos \left(\psi(t)+\psi_{0}\right)}\right) \\
& +\frac{2 p_{\psi} c_{l} I_{l}}{r^{l+1}(t)} \partial_{\psi} P_{l}\left(\sin \iota \sin \left(\psi(t)+\psi_{0}\right)\right)
\end{aligned}
$$

For unbound orbits, one can always integrate Eq. (5.33) to determine $\delta K$. However, for bound periodic orbits there is a possible obstruction: the solution for the conserved quantity $K_{0}+\delta K$ will be single valued if and only if the integral of the source over the closed orbit vanishes,

$$
\oint_{0}^{T_{\mathrm{orb}}} \eta(t) d t=0
$$

Here, $T_{\text {orb }}$ is the orbital period. In other words, the partial differential equation (5.32) has a solution $\delta K$ if and only if the condition (5.35) is satisfied. This is the same condition as obtained by the Poincare-Mel'nikov-Arnold method, a technique for showing the non-integrability and existence of chaos in certain classes of perturbed dynamical systems [35].

Thus, it suffices to show that the condition (5.35) is violated for all multipoles other than the spin and mass quadrupole. To perform the integral in Eq. (5.35), we use the parameterization for the unperturbed motion, $r=$ $K /(1+e \cos \psi)$ and $d t / d \psi=K^{3 / 2} /(1+e \cos \psi)^{2}$, so that the condition for the existence of a conserved quantity $K_{0}+\delta K$ becomes

$$
\int_{0}^{2 \pi} d \psi\left[c_{l} I_{l}(1+e \cos \psi)^{l-1} \partial_{\psi} P_{l}\left(\sin \iota \sin \left(\psi+\psi_{0}\right)\right)-\frac{d_{l} S_{l} L_{z}}{K \sin \iota}(1+e \cos \psi)^{l} \partial_{\psi}\left(\frac{\partial_{\psi} P_{l}\left(\sin \iota \sin \left(\psi+\psi_{0}\right)\right)}{\cos \left(\psi+\psi_{0}\right)}\right)\right]=0
$$

In terms of the variable $\chi=\psi+\psi_{0}-\pi / 2$, Eq. (5.36) can be written as

$$
\begin{aligned}
0 & =\int_{0}^{2 \pi} d \chi c_{l} I_{l}\left[1+e\left(\sin \psi_{0} \cos \chi-\cos \psi_{0} \sin \chi\right)\right]^{l-1} \frac{d}{d \chi} P_{l}(\sin \iota \cos \chi) \\
& +\int_{0}^{2 \pi} d \chi \frac{d_{l} S_{l} L_{z}}{\sin \iota}\left[1+e\left(\sin \psi_{0} \cos \chi-\cos \psi_{0} \sin \chi\right)\right]^{l} \frac{d}{d \chi}\left(\frac{1}{\sin \chi} \frac{d}{d \chi} P_{l}(\sin \iota \cos \chi)\right) .
\end{aligned}
$$

Inserting the expansion (5.28) for $P_{l}(\cos \chi)$, taking the derivatives, and using the binomial expansion for the first term in Eq. (5.37), we get

$$
\begin{aligned}
0 & =c_{l} I_{l} \sum_{n=0}^{N} \sum_{j=0}^{l-1} A_{l n j k} e^{j}(\sin \iota)^{l-2 n}\left(\sin \psi_{0}\right)^{k}\left(\cos \psi_{0}\right)^{j-k} \int_{0}^{2 \pi} d \chi(\sin \chi)^{j-k+1}(\cos \chi)^{k+l-2 n-1} \\
& +\frac{d_{l} S_{l} L_{z}}{K} \sum_{n=0}^{N} \sum_{j=0}^{l} B_{l n j k} e^{j}(\sin \iota)^{l-2 n-1}\left(\sin \psi_{0}\right)^{k}\left(\cos \psi_{0}\right)^{j-k} \int_{0}^{2 \pi} d \chi(\sin \chi)^{j-k+1}(\cos \chi)^{k+l-2 n-2} .
\end{aligned}
$$

The coefficients $A_{l n k j}$ and $B_{l n k j}$ are

$$
A_{l n k j}=\frac{(-1)^{n+k+1}(l-1) !(2 l-2 n) !}{2^{l} n !(l-1-j) ! k !(j-k) !(l-n) !(l-2 n-1) !}, \quad B_{l n k j}=\frac{(-1)^{n+k} l !(2 l-2 n) !}{2^{l} n !(l-j) ! k !(j-k) !(l-n) !(l-2 n-2) !} .
$$

The only non-vanishing contribution to the integrals in Eq. (5.38) will come from terms with even powers of both $\cos \chi$ and $\sin \chi$. These can be evaluated as multiples of the beta function:

$$
\begin{aligned}
0 & =c_{l} I_{l} \sum_{n=0}^{N} \sum_{j=0}^{l-1} C_{l n j k} e^{j}(\sin \iota)^{l-2 n}\left(\sin \psi_{0}\right)^{k}\left(\cos \psi_{0}\right)^{j-k} \delta_{(j-k+1), \text { even }} \delta_{(l+k-1), \text { even }} \\
& +\frac{d_{l} S_{l} L_{z}}{K} \sum_{n=0}^{N} \sum_{j=0}^{l} D_{l n j k} e^{j}(\sin \iota)^{l-2 n-1}\left(\sin \psi_{0}\right)^{k}\left(\cos \psi_{0}\right)^{j-k} \delta_{(j-k+1), \text { even }} \delta_{(l+k), \text { even }}
\end{aligned}
$$


Here, the coefficients are

$$
C_{l n j k}=\frac{2 \Gamma\left(\frac{j}{2}-\frac{k}{2}+1\right) \Gamma\left(\frac{k}{2}+\frac{l}{2}-n\right)}{\Gamma\left(\frac{j}{2}+\frac{l}{2}-n+1\right)} A_{l n k j}, \quad D_{l n j k}=\frac{2 \Gamma\left(\frac{j}{2}-\frac{k}{2}+1\right) \Gamma\left(\frac{k}{2}+\frac{l}{2}-n-\frac{1}{2}\right)}{\Gamma\left(\frac{j}{2}+\frac{l}{2}-n+\frac{3}{2}\right)} B_{l n k j}
$$

Eq. (5.40) shows that for even $l$, terms with $j=$ even (odd) and $k=$ odd (even) give a non-vanishing contribution for the case of a mass (current) multipole, and hence $K_{0}+\delta K$ is not a conserved quantity for the perturbed motion. Note that terms with $j=$ even and $k=$ odd for even $l$ occur only for $l>3$, so for $l=2$ the mass quadrupole term in Eq. (5.40) vanishes and therefore there exists an analog of the Carter constant, which is consistent with our results of Sec. II and our separability analysis. For odd $l$, terms with $j=$ odd (even) and $k=$ even (odd) are finite for $I_{l}\left(S_{l}\right)$. Note that for the case $l=1$ of the spin, the derivatives with respect to $\chi$ in Eq. (5.37) evaluate to zero, so in this case there also exists a Carter-type constant. These results show that for a general multipole other than $I_{2}$ and $S_{1}$, there will not be a Carter-type constant for such a system.

\section{Exact vacuum spacetimes}

Our result on the non-existence of a Carter-type constant can be extended, with mild smoothness assumptions, to falsify the conjecture that all exact, axisymmetric vacuum spacetimes posess a third constant of the motion for geodesic motion. Specifically, we fix a multipole order $l$, and we assume:

- There exists a one parameter family

$$
\left(M, g_{a b}(\lambda)\right)
$$

of spacetimes, which is smooth in the parameter $\lambda$, such that $\lambda=0$ is Schwarzschild, and each spacetime $g_{a b}(\lambda)$ is stationary and axisymmetric with commuting Killing fields $\partial / \partial t$ and $\partial / \partial \phi$, and such that all the mass and current multipole moments of the spacetime vanish except for the one of order $l$. On physical grounds, one expects a one parameter family of metrics with these properties to exist.

- We denote by $H(\lambda)$ the Hamiltonian on the tangent bundle over $M$ for geodesic motion in the metric $g_{a b}(\lambda)$. By hypothesis, there exists for each $\lambda$ a conserved quantity $M(\lambda)$ which is functionally independent of the conserved energy and angular momentum. Our second assumption is that $M(\lambda)$ is differentiable in $\lambda$ at $\lambda=0$. One would expect this to be true on physical grounds.

- We assume that the conserved quantity $M(\lambda)$ is invariant under the symmetries of the system:

$$
\mathcal{L}_{\vec{\xi}} M(\lambda)=\mathcal{L}_{\vec{\eta}} M(\lambda)=0,
$$

where $\vec{\xi}$ and $\vec{\eta}$ are the natural extensions to the 8 dimensional phase space of the Killing vectors $\partial / \partial t$ and $\partial / \partial \phi$. This is a very natural assumption.

These assumptions, when combined with our result of the previous section, lead to a contradiction, showing that the conjecture is false under our assumptions.

To prove this, we start by noting that $M(0)$ is a conserved quantity for geodesic motion in Schwarzschild, so it must be possible to express it as some function $f$ of the three independent conserved quantities:

$$
M(0)=f\left(E, L_{z}, K_{0}\right)
$$

Here $E$ is the energy, $L_{z}$ is the angular momentum, and $K_{0}$ is the Carter constant. Differentiating the exact relation $\{H(\lambda), M(\lambda)\}=0$ and evaluating at $\lambda=0$ gives

$$
\left\{H_{0}, M_{1}\right\}=\frac{\partial f}{\partial E}\left\{E, H_{1}\right\}+\frac{\partial f}{\partial L_{z}}\left\{L_{z}, H_{1}\right\}+\frac{\partial f}{\partial K_{0}}\left\{K_{0}, H_{1}\right\},
$$

where $H_{0}=H(0), H_{1}=H^{\prime}(0)$, and $M_{1}=M^{\prime}(0)$. As before, we can regard this is a partial differential equation that determines $M_{1}$, and a necessary condition for solutions to exist and be single valued is that the integral of the right hand side over any closed orbit must vanish:

$$
\oint\left[\frac{\partial f}{\partial E}\left\{E, H_{1}\right\}+\frac{\partial f}{\partial L_{z}}\left\{L_{z}, H_{1}\right\}+\frac{\partial f}{\partial K_{0}}\left\{K_{0}, H_{1}\right\}\right]=0
$$

Now strictly speaking, there are no closed orbits in the eight dimensional phase space. However, the argument of the previous section applies to orbits which are closed in the four dimensional space with coordinates $\left(r, \theta, p_{r}, p_{\theta}\right)$, since by the third assumption above everything is independent of $t$ and $\phi$, and $p_{t}$ and $p_{\phi}$ are conserved. Here $(t, r, \theta, \phi)$ are Schwarzschild coordinates and $\left(p_{t}, p_{r}, p_{\theta}, p_{\phi}\right)$ are the corresponding conjugate momenta.

Next, we can pull the partial derivatives $\partial f / \partial E$ etc. outside of the integral. It is then easy to see that the first two terms vanish, since there do exist a conserved energy and a conserved $z$-component of angular momentum for the perturbed system. Thus, Eq. (5.44) reduces to

$$
\frac{\partial f}{\partial K_{0}} \oint\left\{K_{0}, H_{1}\right\}=0
$$

Since $M(0)$ is functionally independent of $E$ and $L_{z}$, the prefactor $\partial f / \partial K_{0}$ must be nonzero, so we obtain

$$
\oint\left\{K_{0}, H_{1}\right\}=0 .
$$


The result (5.46) applies to fully relativistic orbits in Schwarzschild. We need to take the Newtonian limit of this result in order to use the result we derived in the previous section. However, the Newtonian limit is a little subtle since Newtonian orbits are closed and generic relativistic orbits are not closed. We now discuss how the limit is taken.

The integral (5.46) is taken over any closed orbit in the four dimensional phase space $\left(r, \theta, p_{r}, p_{\theta}\right)$ which corresponds to a geodesic in Schwarzschild. Such orbits are non generic; they are the orbits for which the ratio between the radial and angular frequencies $\omega_{r}$ and $\omega_{\theta}$ is a rational number. We denote by $q_{r}$ and $q_{\theta}$ the angle variables corresponding to the $r$ and $\theta$ motions [36]. These variables evolve with proper time $\tau$ according to

$$
\begin{aligned}
& q_{r}=q_{r, 0}+\omega_{r} \tau, \\
& q_{\theta}=q_{\theta, 0}+\omega_{\theta} \tau,
\end{aligned}
$$

where $q_{r, 0}$ and $q_{\theta, 0}$ are the initial values. We denote the integrand in Eq. (5.46) by

$$
\mathcal{I}\left(q_{r}, q_{\theta}, a, \varepsilon, \iota\right)
$$

where $\mathcal{I}$ is some function, and $a, \varepsilon$ and $\iota$ are the parameters of the geodesic defined by Hughes 32] (functions of $E, L_{z}$ and $K_{0}$ ). The result (5.46) can be written as

$$
\frac{1}{T} \int_{-T / 2}^{T / 2} d \tau \mathcal{I}\left[q_{r}(\tau), q_{\theta}(\tau), a, \varepsilon, \iota\right]=0,
$$

where $T=T(a, \varepsilon, \iota)$ is the period of the $r, \theta$ motion.

Since the variables $q_{r}$ and $q_{\theta}$ are periodic with period $2 \pi$, we can express the function $\mathcal{I}$ as a Fourier series

$$
\mathcal{I}\left(q_{r}, q_{\theta}, a, \varepsilon, \iota\right)=\sum_{n, m=-\infty}^{\infty} \mathcal{I}_{n m}(a, \varepsilon, \iota) e^{i n q_{r}+i m q_{\theta}}
$$

Now combining Eqs. (5.47), (5.48) and (5.49) gives

$$
\begin{aligned}
0= & \sum_{n, m=-\infty}^{\infty} \mathcal{I}_{n m}(a, \varepsilon, \iota) e^{i n q_{r, 0}+i m q_{\theta, 0}} \\
& \times \operatorname{Si}\left[\left(n \omega_{r}+m \omega_{\theta}\right) T / 2\right]
\end{aligned}
$$

where $\operatorname{Si}(x)=\sin (x) / x$. Since the initial conditions $q_{r, 0}$ and $q_{\theta, 0}$ are arbitrary, it follows that

$$
\mathcal{I}_{n m}(a, \varepsilon, \iota) \operatorname{Si}\left[\left(n \omega_{r}+m \omega_{\theta}\right) T / 2\right]=0
$$

for all $n, m$.

Next, for closed orbits the ratio of the frequencies must be a rational number, so

$$
\frac{w_{r}}{w_{\theta}}=\frac{p}{q}
$$

where $p$ and $q$ are integers with no factor in common. These integers depend on $a, \varepsilon$ and $\iota$. The period $T$ is given by $2 \pi / T=q \omega_{r}=p \omega_{\theta}$. The second factor in Eq. (5.51) now simplifies to

$$
\mathrm{Si}\left[\frac{(n p+m q) \pi}{p q}\right] \text {, }
$$

which vanishes if and only if

$$
n=\bar{n} q, \quad m=\bar{m} p, \quad \bar{n}+\bar{m} \neq 0,
$$

for integers $\bar{n}, \bar{m}$. It follows that

$$
\mathcal{I}_{n m}(a, \varepsilon, \iota)=0
$$

for all $n, m$ except for values of $n, m$ which satisfy the condition (5.54)

Consider now the Newtonian limit, which is the limit $a \rightarrow \infty$ while keeping fixed $\varepsilon$ and $\iota$ and the mass of the black hole. We denote by $\mathcal{I}_{\mathrm{N}}\left(q_{r}, q_{\theta}, a, \varepsilon, \iota\right)$ the Newtonian limit of the function $\mathcal{I}\left(q_{r}, q_{\theta}, a, \varepsilon, \iota\right)$. The integral (5.48) in the Newtonian limit is given by the above computation with $p=q=1$, since $\omega_{r}=\omega_{\theta}$ in this limit. This gives

$$
\frac{1}{T} \oint d \tau \mathcal{I}_{\mathrm{N}}=\sum_{n=-\infty}^{\infty} \mathcal{I}_{\mathrm{N} n,-n}(a, \varepsilon, \iota) e^{i n\left(q_{r, 0}-q_{\theta, 0}\right)}
$$

where $\mathcal{I}_{\mathrm{N} n m}$ are the Fourier components of $\mathcal{I}_{\mathrm{N}}$. In the previous subsection, we showed that this function is nonzero, which implies that there exists a value $k$ of $n$ for which $\mathcal{I}_{\mathrm{N} k,-k} \neq 0$.

Now as $a \rightarrow \infty$, we have $\omega_{r} / \omega_{\theta} \rightarrow 1$, and hence from Eq. (5.52) there exists a critical value $a_{c}$ of $a$ such that the values of $p$ and $q$ exceed $k$ for all closed orbits with $a>a_{c}$. (We are keeping fixed the values of $\varepsilon$ and $\iota$ ). It follows from Eqs. (5.54) and (5.55) that

$$
\frac{\mathcal{I}_{k,-k}(a, \varepsilon, \iota)}{\mathcal{I}_{\mathrm{N} k,-k}(a, \varepsilon, \iota)}=0
$$

for all such values of $a$. However this contradicts the fact that

$$
\frac{\mathcal{I}_{k,-k}(a, \varepsilon, \iota)}{\mathcal{I}_{\mathrm{N} k,-k}(a, \varepsilon, \iota)} \rightarrow 1
$$

as $a \rightarrow \infty$. This completes the proof.

Hence, if the three assumptions listed at the start of this subsection are satisfied, then the conjecture that all vacuum, axisymmetric spacetimes possess a third constant of the motion is false.

Finally, it is sometimes claimed in the classical dynamics literature that perturbation theory is not a sufficiently powerful tool to assess whether the integrability of a system is preserved under deformations. An example that is often quoted is the Toda lattice Hamiltonian [38, 39]. This system is integrable and admits a full set of constants of motion in involution. However, if one approximates the Hamiltonian by Taylor expanding the potential about the origin to third order, one obtains a system which is not integrable. This would seem to indicate 
that perturbation theory can indicate a non-integrability, while the exact system is still integrable.

In fact, the Toda lattice example does not invalidate the method of proof we use here. If we write the Toda lattice Hamiltonian as $H(\mathbf{q}, \mathbf{p})$, then the situation is that $H(\lambda \mathbf{q}, \mathbf{p})$ is integrable for $\lambda=1$, but it is not integrable for $0<\lambda<1$. Expanding $H(\lambda \mathbf{q}, \mathbf{p})$ to third order in $\lambda$ gives a non-integrable Hamiltonian. Thus, the perturbative result is not in disagreement with the exact result for $0<\lambda<1$, it only disagrees with the exact result for $\lambda=1$. In other words, the example shows that perturbation theory can fail to yield the correct result for finite values of $\lambda$, but there is no indication that it fails in arbitrarily small neighborhoods of $\lambda=0$. Our application is qualitatively different from the Toda lattice example since we have a one parameter family of Hamiltonians $H(\lambda)$ which by assumption are integrable for all values of $\lambda$.

\section{CONCLUSION}

We have examined the effect of an axisymmetric quadrupole moment $Q$ of a central body on test particle inspirals, to linear order in $Q$, to the leading postNewtonian order, and to linear order in the mass ratio. Our analysis shows that a natural generalization of the Carter constant can be defined for the quadrupole interaction. We have also analyzed the leading order spin selfinteraction effect due to the scattering of the radiation off the spacetime curvature due to the spin. Combining the effects of the quadrupole and the leading order effects linear and quadratic in the spin, we have obtained expressions for the instantaneous as well as time-averaged evolution of the constants of motion for generic orbits under gravitational radiation reaction, complete at $O\left(a^{2} \epsilon^{4}\right)$. We have also shown that for a single multipole interaction other than $Q$ or spin, in our approximations, a Cartertype constant does not exist. With mild additional assumptions, this result can be extended to exact spacetimes and falsifies the conjecture that all axisymmetric vacuum spacetimes possess a third constant of motion for geodesic motion.

\section{ACKNOWLEDGMENTS}

This research was partially supported by NSF grant PHY-0457200. We thank Jeandrew Brink for useful correspondence.

\section{Appendix A: Time variation of quadrupole: order of} magnitude estimates

In this appendix, we give an estimate of the timescale $T_{\text {evol }}$ for the quadrupole to change. The analysis in the body of this paper is valid only when $T_{\text {evol }} \gg T_{\text {rr }}$, where
$T_{r r}$ is the radiation reaction time, since we have neglected the time evolution of the quadrupole. We distinguish between two cases: (i) when the central body is exactly nonspinning but has a quadrupole, and (ii) when the central body has finite spin in addition to the quadrupole.

\section{Estimate of the scaling for the nonspinning case}

For the purpose of a crude estimate, the relevant interaction is the tidal interaction with energy

$$
Q_{i j} \mathcal{E}_{i j} \sim-\frac{m_{2}}{r^{3}} \bar{Q} I \cos ^{2} \theta,
$$

where $\mathcal{E}_{i j}$ is the tidal field, $\theta$ is the angle between the symmetry axis and the normal to the orbital plane of $m_{2}$, and we have written the quadrupole as $Q \sim \bar{Q} I$, where $\bar{Q}$ is dimensionless and $I$ is the moment of inertia. For small deviations from equilibrium, the relevant piece of the Lagrangian is schematically

$$
L \sim I \dot{\psi}^{2}+\bar{Q} I \frac{m_{2}}{r^{3}} \psi^{2} .
$$

We define the evolution timescale $T_{\text {evol }}$ to be the time it takes for the angle to change by an amount of order unity, and since the amplitude of the oscillation scales roughly as $\sim m_{2} / m_{1}$, the evolution time scales as

$$
T_{\mathrm{evol}}^{-2} \sim \frac{m_{2}^{2}}{m_{1}^{2}} \bar{Q}\left(\frac{m_{2}}{M}\right) \omega_{\mathrm{orbit}}^{2},
$$

where $\omega_{\text {orbit }}^{2}=M / r^{3}$. Thus, the ratio of the evolution timescale compared to the radiation reaction timescale scales as

$$
T_{\text {evol }} / T_{\mathrm{rr}} \sim(1 / \sqrt{\bar{Q}}) \frac{m_{1}}{m_{2}}\left(\frac{\mu}{M}\right)^{1 / 2}\left(\frac{M}{r}\right)^{5 / 2} .
$$

\section{Estimate of the scaling for the spinning case}

When the body is spinning the effect of the tidal coupling is to cause a precession. For the purpose of this estimate, we calculate the torque on $m_{1}$ due to the companion's Newtonian field. The torque $\mathbf{N}$ scales as

$$
N_{i} \sim \epsilon_{i m j} Q_{m k} \mathcal{E}_{j k} .
$$

We assume that the precession is slow, i.e.

$$
\omega_{\text {prec }} \ll \bar{S} / m_{1}\left(\frac{m_{2}}{M}\right),
$$

where $\omega_{\text {prec }}$ is the precession frequency and $\bar{S}=S / m_{1}^{2}$ is the dimensionless spin. This gives the approximate scaling of the precession timescale as (cf. [37])

$$
T_{\text {prec }} / T_{\text {rr }} \sim \frac{\bar{S}}{\bar{Q}}\left(\frac{M}{r}\right) .
$$


and the evolution timescale is thus

$$
T_{\mathrm{evol}} / T_{\mathrm{rr}} \sim \frac{m_{1}}{m_{2}} \frac{\bar{S}}{\bar{Q}}\left(\frac{M}{r}\right) .
$$

Because of our assumption (A6) that the precession is slow, equation (A8) is valid only when

$$
1 \gg\left(\frac{\mu}{M}\right) \frac{\bar{S}^{2}}{\bar{Q}}\left(\frac{r}{M}\right)^{3} .
$$

When $\bar{S}$ is sufficiently small that the condition (A9) is violated, the relevant timescale is instead given by Eq. (A3).

\section{Application to Kerr inspirals}

For Kerr inspirals,

$$
\bar{S} \sim a, \quad \bar{Q} \sim a^{2}, \quad \mu / M \ll 1 \text { and } r \sim M .
$$

Therefore, the condition A9 is satisfied, and the precession time is longer than the radiation reaction time by

$$
T_{\text {prec }} / T_{\text {rr }} \sim \frac{1}{a}\left(\frac{M}{r}\right) .
$$

Note that for Kerr inspirals, since $r \sim M$ both formulas (A3) and (A7) give the same scaling.

Moreover, for Kerr inspirals, the amplitude of the precession will be small, of order the mass ratio $\mu / M$. This is because of angular momentum conservation: in the relativistic regime, the orbital angular momentum is a factor of $\mu / M$ smaller than the angular momentum of the black hole and can therefore not cause a large precession amplitude. Even if the orbital angular momentum at infinity is large, most of it will be radiated away as outgoing gravitational waves during the earlier phase of the inspiral. This factor of $\mu / M$ is taken into account when we consider the evolution timescale, which for Kerr inspirals reduces to

$$
T_{\text {evol }} / T_{\mathrm{rr}} \sim\left(\frac{M}{\mu}\right)\left(\frac{1}{a}\right)\left(\frac{M}{r}\right) .
$$

Since $1 / a \geq 1, M / r \sim 1$ and $M / \mu \ll 1$, the evolution time is long compared to the radiation reaction time and we can neglect the time variation of the quadrupole at leading order.

\section{Appendix B: Computation of time averaged fluxes}

\section{Averaging method that parallels fully relativistic averaging}

We start by noting that the differential equations (2.26) and (2.27) governing the $\tilde{r}$ and $\tilde{\theta}$ motions decouple if we define a new time parameter $\hat{t}$ by

$$
d \hat{t}=\frac{1}{\tilde{r}^{2}} d \tilde{t}
$$

This is the analog of the Mino time parameter for geodesic motion in Kerr [12]. The equations of motion (2.26) -2.24) then become

$$
\begin{aligned}
\left(\frac{d \tilde{r}}{d \hat{t}}\right)^{2}= & \hat{V}_{\tilde{r}}(\tilde{r}), \\
\hat{V}_{\tilde{r}}(\tilde{r})= & 2 E \tilde{r}^{4}+2 \tilde{r}^{3}-K \tilde{r}^{2}-4 S L_{z} \tilde{r} \\
& +\frac{Q}{2}\left(\tilde{r}-2 L_{z}^{2}\right), \\
\left(\frac{d \tilde{\theta}}{d \hat{t}}\right)^{2}= & \hat{V}_{\tilde{\theta}}(\tilde{\theta}), \\
\hat{V}_{\tilde{\theta}}(\tilde{\theta})= & K-\frac{L_{z}^{2}}{\sin ^{2} \tilde{\theta}}-Q E \cos 2 \tilde{\theta} \\
\left(\frac{d \varphi}{d \hat{t}}\right)= & \hat{V}_{\varphi \tilde{r}}(\tilde{r})+\hat{V}_{\varphi \tilde{\theta}}(\tilde{\theta}), \\
\hat{V}_{\varphi \tilde{r}}(\tilde{r})= & \frac{Q L_{z}}{\tilde{r}^{2}}, \quad \hat{V}_{\varphi \tilde{\theta}}(\tilde{\theta})=\frac{L_{z}}{\sin ^{2} \tilde{\theta}} .
\end{aligned}
$$

The parameters $t$ and $\hat{t}$ are related by:

$$
\begin{aligned}
\frac{d t}{d \hat{t}} & =\hat{V}_{t \tilde{r}}(\tilde{r})+\hat{V}_{t \tilde{\theta}}(\tilde{\theta}) \\
\hat{V}_{t \tilde{r}}(\tilde{r}) & =\tilde{r}^{2}, \quad \hat{V}_{t \tilde{\theta}}(\tilde{\theta})=\frac{Q}{2} \cos 2 \tilde{\theta} .
\end{aligned}
$$

It follows from Eqs. (B2) and (B4) that the functions $\tilde{r}(\hat{t})$ and $\tilde{\theta}(\hat{t})$ are periodic; and we denote their periods by $\Lambda_{\tilde{r}}$ and $\Lambda_{\tilde{\theta}}$. We define the fiducial motion associated with the constants of motion $E, L_{z}$ and $K$ to be the motion with the initial conditions $\tilde{r}(0)=\tilde{r}_{\min }$ and $\tilde{\theta}(0)=$ $\tilde{\theta}_{\min }$, where $\tilde{r}_{\min }$ and $\tilde{\theta}_{\text {min }}$ are given by the vanishing of the right-hand sides of Eqs. (B2) and (B4) respectively. The functions $\hat{r}(\hat{t})$ and $\hat{\theta}(\hat{t})$ associated with this fiducial motion are given by

$$
\begin{gathered}
\int_{\tilde{r}_{\min }}^{\hat{r}(\hat{t})} \frac{d \tilde{r}}{ \pm \sqrt{\hat{V}_{\tilde{r}}(\tilde{r})}}=\hat{t}, \\
\int_{\tilde{\theta}_{\min }}^{\hat{\theta}(\hat{t})} \frac{d \tilde{\theta}}{ \pm \sqrt{\hat{V}_{\tilde{\theta}}(\tilde{\theta})}}=\hat{t} .
\end{gathered}
$$

From Eq. (B8) it follows that

$$
t(\hat{t})=t_{0}+\int_{0}^{\hat{t}} d t^{\prime}\left(\hat{V}_{t \tilde{r}}\left[\tilde{r}\left(t^{\prime}\right)\right]+\hat{V}_{t \tilde{\theta}}\left[\tilde{\theta}\left(t^{\prime}\right)\right]\right),
$$

where $t_{0}=t(0)$. Next, we define the constant $\Gamma$ to be the following average value:

$$
\Gamma=\frac{1}{\Lambda_{\tilde{r}}} \int_{0}^{\Lambda_{\tilde{r}}} d t^{\prime} \hat{V}_{t \tilde{r}}\left[\hat{r}\left(t^{\prime}\right)\right]+\frac{1}{\Lambda_{\tilde{\theta}}} \int_{0}^{\Lambda_{\tilde{\theta}}} d t^{\prime} \hat{V}_{t \tilde{\theta}}\left[\hat{\theta}\left(t^{\prime}\right)\right]
$$


Then we can write $t(\hat{t})$ as a sum of a linear term and terms that are periodic:

$$
t(t)=t_{0}+\Gamma \hat{t}+\delta t(\hat{t}),
$$

where $\delta t(\hat{t})$ denotes the oscillatory terms in Eq. (B12).

To average a function over the time parameter $\hat{t}$, it is convenient to parameterize $\tilde{r}$ and $\tilde{\theta}$ in terms of angular variables as follows. For the average over $\tilde{\theta}$ we introduce the parameter $\chi$ by

$$
\cos ^{2} \hat{\theta}(\hat{t})=z_{-} \cos ^{2} \chi
$$

where $z_{-}=\cos ^{2} \tilde{\theta}_{-}$with $z_{-}$being the smaller root of Eq. (B4):

$$
z_{ \pm}=\frac{1}{2 \beta}\left[K+3 Q E \pm \sqrt{(K-Q E)^{2}+4 Q E L_{z}^{2}}\right]
$$

and where $\beta=2 Q E$. Then from the definition (B11) of $\hat{\theta}$ together with Eq. (B4) and the requirement that $\chi$ increases monotonically with $\hat{t}$ we obtain

$$
\frac{d \chi}{d \hat{t}}=\sqrt{\beta\left(z_{+}-z_{-} \cos ^{2} \chi\right)}
$$

Then we can write the average over $\hat{t}$ of a function $F_{\tilde{\theta}}(\hat{t})$ which is periodic with period $\Lambda_{\tilde{\theta}}$ in terms of $\chi$ as

$$
\begin{aligned}
\left\langle F_{\tilde{\theta}}\right\rangle_{\hat{t}} & =\frac{1}{\Lambda_{\tilde{\theta}}} \int_{0}^{\Lambda_{\tilde{\theta}}} d \hat{t} F_{\tilde{\theta}}(\hat{t}) \\
& =\frac{1}{\Lambda_{\tilde{\theta}}} \int_{0}^{2 \pi} d \chi \frac{F_{\tilde{\theta}}[\hat{t}(\chi)]}{\sqrt{\beta\left(z_{+}-z_{-} \cos ^{2} \chi\right)}}
\end{aligned}
$$

where

$$
\Lambda_{\tilde{\theta}}=\int_{0}^{2 \pi} d \chi \frac{1}{\sqrt{\beta\left(z_{+}-z_{-} \cos ^{2} \chi\right)}} .
$$

Similarly, to average a function $F_{\tilde{r}}(\hat{t})$ that is periodic with period $\Lambda_{\tilde{r}}$, we introduce a parameter $\xi$ via

$$
\tilde{r}=\frac{p}{1+e \cos \xi}
$$

where the parameter $\xi$ varies from 0 to $2 \pi$ as $\tilde{r}$ goes through a complete cycle. Then,

$$
\begin{aligned}
\frac{d \xi}{d \hat{t}} & =P(\xi) \\
P(\xi) & \equiv\left(\hat{V}_{\tilde{r}}[\tilde{r}(\xi)]\right)^{1 / 2}\left[\frac{p e|\sin \xi|}{(1+e \cos \xi)^{2}}\right]^{-1}
\end{aligned}
$$

The average over $\hat{t}$ of $F_{\tilde{r}}(\hat{t})$ can then be computed from

$$
\left\langle F_{\tilde{r}}\right\rangle_{\hat{t}}=\frac{\int_{0}^{2 \pi} d \xi F_{\tilde{r}} / P(\xi)}{\int_{0}^{2 \pi} d \xi / P(\xi)} .
$$

Now, a generic function $F_{\tilde{r}, \tilde{\theta}}[\tilde{r}(\hat{t}), \tilde{\theta}(\hat{t})]$ will be biperiodic in $\hat{t}: F_{\tilde{r}, \tilde{\theta}}\left[\tilde{r}\left(\hat{t}+\Lambda_{\tilde{r}}\right), \tilde{\theta}\left(\hat{t}+\Lambda_{\tilde{\theta}}\right)\right]=F_{\tilde{r}, \tilde{\theta}}[\tilde{r}(\hat{t}), \tilde{\theta}(\hat{t})]$. Combining the results (B18) and (B23) we can write its average as a double integral over $\chi$ and $\xi$ as

$\left\langle F_{\tilde{r}, \tilde{\theta}}\right\rangle_{\hat{t}}=\frac{1}{\Lambda_{\tilde{\theta}} \Lambda_{\tilde{r}}} \int_{0}^{2 \pi} d \chi \int_{0}^{2 \pi} d \xi \frac{F_{\tilde{r}, \tilde{\theta}}[\tilde{r}(\xi), \tilde{\theta}(\chi)]}{\sqrt{\beta\left(z_{+}-z_{-} \cos ^{2} \chi\right)} P(\xi)}$.

To compute the time average of $\dot{E}, \dot{L}_{z}$, and $\dot{K}$, we need to convert the average of a function over $\hat{t}$ calculated from (B24) to the average over $t$. As explained in detail in [9], in the adiabatic limit we can choose a time interval $\Delta t$ which is long compared to the orbital timescale but short compared to the radiation reaction time. From Eq. (B12) we have $\Delta t=\Gamma \hat{t}+$ osc.terms. The oscillatory terms will be bounded and will therefore be negligible in the adiabatic limit, so we have to a good approximation

$$
\langle\dot{E}\rangle_{t}=\frac{1}{\Gamma}\left\langle\dot{E} \hat{V}_{t}\right\rangle_{\hat{t}}
$$

where $\hat{V}_{t} \equiv \hat{V}_{t \tilde{r}}+\hat{V}_{t \tilde{\theta}}$, cf. Eq. (B8), and similarly for $\dot{L}_{z}$ and $\dot{K}$.

The explicit results we obtain using this method are given in section III, Eqs. (3.28), (3.29), and (3.30).

\section{Averaging method using the explicit parameterization of Newtonian orbits}

To perform the time-averaging using this method, we define a parameter $\xi$ via

$$
\tilde{r}=\frac{p}{1+e \cos \xi}
$$

where the parameter $\xi$ varies from 0 to $2 \pi$ as $\tilde{r}$ goes through a complete cycle. Note that $\theta$ appears in Eqs. (3.16) - 3.18) only in terms that are linear in $Q$, so we can write $\theta$ in terms of $\xi$ using the Newtonian relation

$$
x_{3}=r \cos \theta=r \sin \iota \sin \left(\xi+\xi_{0}\right) \text {. }
$$

Here, $\xi_{0}$ is the angle between the direction of the perihelion and the intersection of the orbital and equatorial plane. Similarly, for the $\dot{r} \dot{\theta}$ terms in Eqs. (3.17) and (3.26) we can use the Newtonian relations $\dot{r}=e / \sqrt{p} \sin \xi$ and $\dot{\xi}=\sqrt{p} / r^{2}$. From Eqs. (2.27) and (B20) it follows that 


$$
\frac{d \tilde{t}}{d \xi}=\frac{p^{3 / 2}}{(1+e \cos \xi)^{2}}\left\{1-\frac{Q}{8 p^{2}}\left[-3+e^{2}-2 e \cos \xi+2 \cos ^{2} \iota\left(8-e^{2}+8 e \cos \xi+e^{2} \cos 2 \xi\right)\right]\right\}
$$

and from Eq. (2.12)

$$
\frac{d t}{d \tilde{t}}=\left\{1+\frac{Q}{2 p^{2}}(1+e \cos \xi)\left[2 \sin ^{2} \iota \sin ^{2}\left(\xi+\xi_{0}\right)-1\right]\right\}
$$

Using these expressions, we compute the time-averaged fluxes from

$$
\langle\dot{E}\rangle=\frac{\int_{0}^{2 \pi} d \xi \dot{E}(d t / d \tilde{t})(d \tilde{t} / d \xi)}{\int_{0}^{2 \pi} d \xi(d t / d \tilde{t})(d \tilde{t} / d \xi)}
$$

and obtain:

$$
\begin{aligned}
& \langle\dot{E}\rangle=-\frac{32}{5} \frac{\left(1-e^{2}\right)^{3 / 2}}{p^{5}} \quad\left[1+\frac{73}{24} e^{2}+\frac{37}{96} e^{4}-\frac{S}{p^{3 / 2}}\left(\frac{73}{12}+\frac{823}{24} e^{2}+\frac{949}{32} e^{4}+\frac{491}{192} e^{6}\right) \cos (\iota)\right. \\
& -\frac{Q}{p^{2}}\left\{\frac{1}{2}+\frac{85}{32} e^{2}+\frac{349}{128} e^{4}+\frac{107}{384} e^{6}+\left(\frac{11}{4}+\frac{273}{16} e^{2}+\frac{847}{64} e^{4}+\frac{179}{192} e^{6}\right) \cos (2 \iota)\right\} \\
& -\frac{S^{2}}{p^{2}}\left\{\frac{13}{192}+\frac{247}{384} e^{2}+\frac{299}{512} e^{4}+\frac{39}{1024} e^{6}-\left(\frac{1}{192}+\frac{19}{384} e^{2}+\frac{23}{512} e^{4}+\frac{3}{1024} e^{6}\right) \cos (2 \iota)\right\} \\
& -\frac{Q}{p^{2}} e^{2}\left(\frac{869}{48}+\frac{1595}{96} e^{2}+\frac{121}{128} e^{4}\right) \cos \left(2 \xi_{0}\right) \sin ^{2} \iota \\
& \left.+\frac{S^{2}}{p^{2}} e^{2}\left(\frac{1}{384}+\frac{5}{384} e^{2}+\frac{3}{2084} e^{4}\right) \cos \left(2 \xi_{0}\right) \sin ^{2} \iota\right] \\
& \left\langle\dot{L}_{z}\right\rangle=-\frac{32}{5} \frac{\left(1-e^{2}\right)^{3 / 2}}{p^{7 / 2}} \cos \iota \quad\left[1+\frac{7}{8} e^{2}-\frac{S}{2 p^{3 / 2} \cos \iota}\left\{\frac{61}{24}+7 e^{2}+\frac{271}{64} e^{4}+\left(\frac{61}{8}+\frac{91}{4} e^{2}+\frac{461}{64} e^{4}\right) \cos (2 \iota)\right\}\right. \\
& -\frac{Q}{16 p^{2}}\left\{-3-\frac{45}{4} e^{2}+\frac{19}{8} e^{4}+\left(45+148 e^{2}+\frac{331}{8} e^{4}\right) \cos (2 \iota)\right\} \\
& \left.+\frac{S^{2}}{16 p^{2}}\left\{1+3 e^{2}+\frac{3}{8} e^{4}\right\}-\frac{Q}{p^{2}} e^{2} \cos \left(2 \xi_{0}\right) \sin ^{2} \iota\left(\frac{201}{32}+\frac{51}{32} e^{2}\right)\right], \\
& \langle\dot{K}\rangle=-\frac{64}{5} \frac{\left(1-e^{2}\right)^{3 / 2}}{p^{3}} \quad\left[1+\frac{7}{8} e^{2}-\frac{S}{2 p^{3 / 2}}\left(\frac{97}{6}+37 e^{2}+\frac{211}{16} e^{4}\right) \cos (\iota)\right. \\
& -\frac{Q}{p^{2}}\left\{\frac{1}{2}+\frac{55}{48} e^{2}+\frac{139}{192} e^{4}+\left(\frac{13}{4}+\frac{841}{96} e^{2}+\frac{449}{192} e^{4}\right) \cos (2 \iota)\right\} \\
& +\frac{S^{2}}{p^{2}}\left\{\frac{13}{192}+\frac{13}{64} e^{2}+\frac{13}{512} e^{4}-\left(\frac{1}{192}+\frac{1}{64} e^{2}+\frac{1}{512} e^{4}\right) \cos (2 \iota)\right\} \\
& \left.-\frac{Q}{p^{2}}\left(\frac{391}{48}+\frac{37}{24} e^{2}\right) e^{2} \cos \left(2 \xi_{0}\right) \sin ^{2} \iota\right] \text {. }
\end{aligned}
$$

In the adiabatic limit, the terms involving $\cos \left(2 \xi_{0}\right)$ can be omitted because they average to zero. As explained by Ryan [15], the radiation reaction timescale for terms involving $\xi_{0}$ is much longer than the precession timescale for most orbits, so the terms involving $\xi_{0}$ will average away. This is consistent with our results for the adia- batic infinite time-averaged fluxes using the Mino time parameter. The Mino-time averaging method was based on the assumption that the fundamental frequencies are incommensurate and the motion fills up the whole torus, which is equivalent to averaging over $\xi_{0}$. 
23, 4167 (2006)

[3] D. A. Brown, et al. gr-qc/0612060

[4] E. Poisson, Living Rev. Relat. 7, 6 (2004), http://relativity.livingreviews.org/Articles/lrr-2004-6/ind

[5] Special Issue: Gravit. Rad. from Binary Black Holes: Advances in the perturbative approach, Class. Quant. Grav. $22(2005)$

[6] K. Glampedakis, Class. Quantum Grav. 22, S605 (2005)

[7] S. Drasco, Class. Quantum Grav. 23, S769 (2006)

[8] M. Favata and É. É. Flanagan Accuracy of adiabatic waveforms for eccentric orbits, (in preparation)

[9] S. Drasco, É. É. Flanagan, and S. A. Hughes, Class. Quantum Grav. 22, S801 (2005)

[10] É. É. Flanagan and T. Hinderer, Two timescale analysis of extreme mass ratio inspirals in Kerr. II. Numerical integration through resonances, (in preparation)

[11] N. Sago, et al., Progr. Theor. Phys. 114, 509 (2005)

[12] Y. Mino, Phys. Rev. D 67, 084027 (2003)

[13] S. A. Hughes, S. Drasco, É. É. Flanagan, and J. Franklin, Phys. Rev. Lett. 94, 221101 (2005)

[14] F. D. Ryan, Phys. Rev. D 52, R3159 (1995)

[15] F. D. Ryan, Phys. Rev. D 53, 3064 (1996)

[16] L. Blanchet, T. Damour, G. Farese, and B. Iyer, Phys. Rev. D 71, 124004 (2005)

[17] L. Kidder, C. Will, and A. Wiseman, Phys. Rev. D 47, R4183 (1993)

[18] L. A. Gergely, Phys. Rev. D 61, 024035 (1999)

[19] C. Will, Phys. Rev. D 71, 084027 (2005)

[20] G. Faye, L. Blanchet, and A. Buonanno, Phys. Rev. D 74, 104033 (2006)

[21] L. Blanchet, A. Buonanno, and G. Faye, Phys. Rev. D
74, 104034 (2006)

[22] E. Poisson, Phys. Rev. D 57, 5287 (1998)

[23] L. A. Gergely and Z. Keresztes, Phys. Rev. D 67, 024020 html (2003)

[24] M. Shibata, M. Sasaki, H. Tagoshi, and T. Tanaka, Phys. Rev. D 51, 1646 (1995)

[25] É. É. Flanagan and T. Hinderer, Two timescale analysis of extreme mass ratio inspirals in Kerr. I. General formalism, (in preparation)

[26] C. Misner, K. Thorne, and J. Wheeler, Gravitation (W.H. Freeman and Co., San Francisco, 1973)

[27] R. O. Hansen, J. Math. Phys. 15, 46 (1974)

[28] K. S. Thorne, Rev. Mod. Phys 52, 300 (1980)

[29] T. Bäckdahl, gr-qc/0612043 (2006); Class. Quantum Grav. 22, 3585 (2005)

[30] C. Li and G. Lovelace, gr-qc/0702146 (2007)

[31] L. Blanchet and T. Damour, Phys. Lett. 104A, 82 (1984)

[32] S. A. Hughes, Phys. Rev. D 61, 084004 (2000)

[33] K. Glampedakis, S. Hughes, and D. Kennefick, Phys. Rev. D 66, 064005 (2002)

[34] G. Arfken, Mathematical Methods for Physicists (Academic Press, CA, 1985)

[35] V. K. Melnikov, Trans. Moscow Math. Soc. 12, 1-56 (1956)

[36] W. Schmidt, Celestial mechanics in Kerr spacetime Class. Quantum Grav. 19 (2002) 2743-2764

[37] H. Goldstein, C. Poole, and J. Safko, Classical Mechanics (Addison-Wesley, 2002)

[38] Ý. Birol and A. Hacinliyan, Phys. Rev. E 52, 4750 (1995)

[39] H. Yoshida, Comm. Math. Phys. 116, 529 (1988) 